\title{
Diagnosis of tuberculosis in wildlife: a systematic review
}

\author{
Jobin Thomas ${ }^{1,2}$, Ana Balseiro ${ }^{3,4^{*}}$ (D) , Christian Gortázar ${ }^{1}$ and María A. Risalde 5,6
}

\begin{abstract}
Animal tuberculosis (TB) is a multi-host disease caused by members of the Mycobacterium tuberculosis complex (MTC). Due to its impact on economy, sanitary standards of milk and meat industry, public health and conservation, TB control is an actively ongoing research subject. Several wildlife species are involved in the maintenance and transmission of TB, so that new approaches to wildlife TB diagnosis have gained relevance in recent years. Diagnosis is a paramount step for screening, epidemiological investigation, as well as for ensuring the success of control strategies such as vaccination trials. This is the first review that systematically addresses data available for the diagnosis of TB in wildlife following the Preferred Reporting Items of Systematic Reviews and Meta-Analyses (PRISMA) guidelines. The article also gives an overview of the factors related to host, environment, sampling, and diagnostic techniques which can affect test performance. After three screenings, 124 articles were considered for systematic review. Literature indicates that post-mortem examination and culture are useful methods for disease surveillance, but immunological diagnostic tests based on cellular and humoral immune response detection are gaining importance in wild life TB diagnosis. Among them, serological tests are especially useful in wild life because they are relatively inexpensive and easy to perform, facilitate large-scale surveillance and can be used both ante- and post-mortem. Currently available studies assessed test performance mostly in cervids, European badgers, wild suids and wild bovids. Research to improve diagnostic tests for wild life TB diagnosis is still needed in order to reach accurate, rapid and cost-effective diagnostic techniques adequate to a broad range of target species and consistent over space and time to allow proper disease monitoring.
\end{abstract}

Keywords: animal tuberculosis, diagnosis, immunological methods, Mycobacterium tuberculosis complex, PRISMA, systematic review, wildlife

\section{Table of Contents}

Introduction

Overview and importance of wild hosts

Relevance of diagnosis in wildlife

Methods

Results and discussion

Principal methods of TB diagnosis

TB like lesions (TBL)

*Correspondence: abalm@unileon.es

${ }^{3}$ Departamento de Sanidad Animal, Facultad de Veterinaria, Universidad de León, 24071 León, Spain

Full list of author information is available at the end of the article
Post-mortem examination

Histopathological examination

Identification of the microorganism

Microscopy.

Microbiological culture

Molecular identification

Tests based on immune response

CMI based diagnostics.

Other tests

Confounding factors

Host

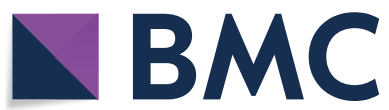

(c) The Author(s) 2021. This article is licensed under a Creative Commons Attribution 4.0 International License, which permits use, sharing, adaptation, distribution and reproduction in any medium or format, as long as you give appropriate credit to the original author(s) and the source, provide a link to the Creative Commons licence, and indicate if changes were made. The images or other third party material in this article are included in the article's Creative Commons licence, unless indicated otherwise in a credit line to the material. If material is not included in the article's Creative Commons licence and your intended use is not permitted by statutory regulation or exceeds the permitted use, you will need to obtain permission directly from the copyright holder. To view a copy of this licence, visit http://creativeco mmons.org/licenses/by/4.0/. The Creative Commons Public Domain Dedication waiver (http://creativecommons.org/publicdomain/ zero/1.0/) applies to the data made available in this article, unless otherwise stated in a credit line to the data. 
Environment, habitat and management

Prior sensitization, history and other infections

Sample and sampling related factors

Diagnostic technique related factors

Improved diagnosis

Selection of the appropriate test

Proper implementation and interpretation

of the test

Combination of different diagnostic tests or different antigens

Conclusions

References

\section{Introduction}

\section{Overview and importance of wild hosts}

Animal tuberculosis (TB) is a globally distributed disease caused by members of the Mycobacterium tuberculosis complex (MTC), which can infect humans and a broad range of domestic and wild mammals [1]. TB is a highly relevant zoonosis, causing risk to public health and financial loss due to decreased production, obligatory slaughter of test-positive animals as well as cost of preventive measures. Moreover, it causes threat to conservation strategies in and around protected natural areas [1,2]. Several wildlife species act as maintenance host, spill over host or host with unknown reservoir status depending on the region. In Europe, Eurasian wild boar (Sus scrofa) (Iberian Peninsula), red deer (Cervus elaphus) (Iberian Peninsula, Western Austria), fallow deer (Dama dama) (Iberian Peninsula) and European badger (Meles meles) (British Isles and Atlantic Spain) are regarded as main wildlife MTC reservoir hosts [2-5]. In Africa, wildlife reservoir hosts include common warthog (Phacochoerus africanus) (South Africa), African buffalo (Syncerus caffer) (South Africa), lechwe antelope (Kobus leche) (South Africa) and Eurasian wild boar (North Africa) [6-8]. In addition, wild meerkats (Suricata suricatta) (South Africa), African elephant (Loxodonta africana) (South Africa), white rhinoceros (Ceratotheriumsimum) (South Africa), Nyala (Tragelaphus angasii) (South Africa), African lion (Panthera leo) (South Africa) and banded mongooses (Mungos mungo) (South Africa) are frequently affected with TB [9-13]. In North America, white-tailed deer (Odocoileus virginianus) (Michigan, Minnesota, Mexico), wood bison (Bison bison) (Canada) and elk (Cervus canadensis) (Canada) are the major wildlife hosts identified [14]. In South America, the information with regard to wildlife TB is scarce, even though there are some implications that Brazilian wild boar (Sus scrofa) (Brazil) plays a role as disease reservoir [15]. In
New Zealand, the Australian brushtail possum (Trichosurus vulpecula) acts as primary wildlife reservoir host [16]. In Asia, the disease has been reported in many wild animal species, but there is considerable research gap on this area regarding the role of wildlife in MTC epidemiology. Generally, the above-mentioned species are the potential reservoir hosts in different continents. However, in some regions, disease is on the verge of eradication or infection rate has been considerably reduced due to the intensive diagnosis and prevention protocols [17].

\section{Relevance of diagnosis in wildlife}

Diagnosis in wildlife is (i) a prime step in disease control and management [18], but is also essential (ii) in the evaluation of surveillance strategies, (iii) in pathogenesis, epidemiological and transmission studies as well as (iv) in the assessment of the efficacy of vaccination trials [1820]. However, diagnosis in wildlife is challenging due to the wide taxonomic diversity, the capture and restraint difficulties inherent to wildlife collection of samples, frequent lack of gold standard diagnostic techniques, lack of knowledge about the true infection status, difficulty in interpretation and conducting experimental studies, as well as limited financial resources [18]. Nevertheless, TB in wildlife is an active area of research.

Many studies have been carried out in order to overcome the problems associated with TB testing and surveillance in wildlife. The lines of investigations include development of new diagnostic techniques like rapid tests [STAT PAK assay or Dual Path Platform (DPP) tests] $[10,21]$ or the modification of existing ones [21-25] in order to improve the diagnostic efficiency and accuracy in wildlife while remaining practical. Some reviews have addressed TB diagnosis in domestic animals and wildlife, underlining TB diagnostic techniques in the context of disease control and eradication [26-30], identifying some research gaps as well as the need of more reliable approaches for TB diagnosis in wildlife. The present work is the first review that summarizes data available from the currently applicable techniques for TB diagnosis in wildlife, especially highlighting the immunological methods, by means of a systematic system following the Preferred Reporting Items of Systematic Reviews and Meta-Analyses (PRISMA) guidelines. We find that, while culture remains as the gold standard method despite its limited sensitivity (Se), there has been considerable progress in cellular and humoral immunological diagnostic tests for wildlife TB diagnosis. Serological tests are especially useful in wildlife because they are economically attractive, technically easy, enable large-scale surveillance and can be applied both in live or dead animals and, in the latter, in combination with pathology. In farmed wildlife, combinations of cellular and humoral tests could enhance diagnostic accuracy. 


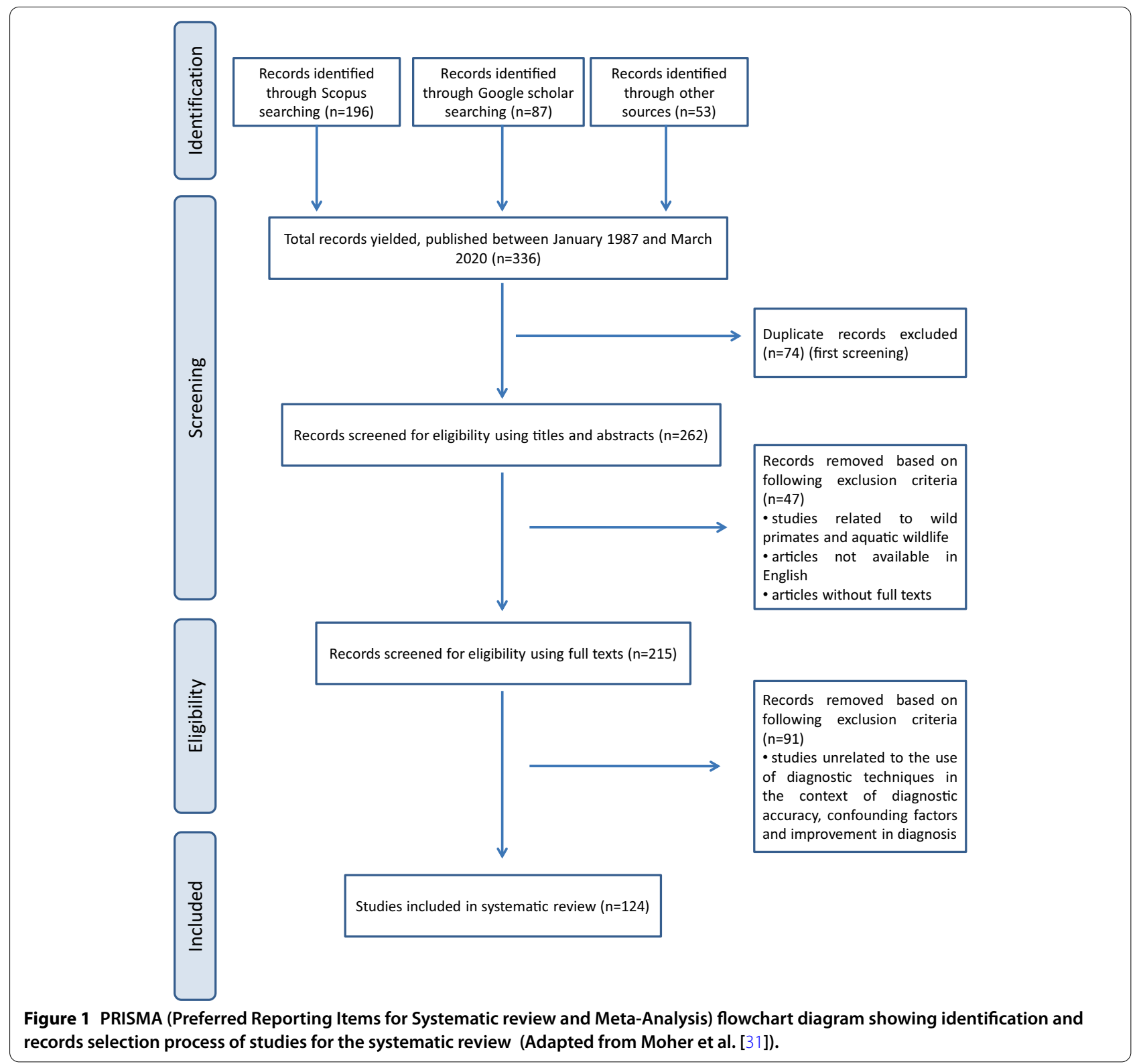

\section{Methods}

This study followed the PRISMA recommendations for systematic review reporting [31]. The research question was: which are the studies available for diagnosis of TB in wild mammals, the influence of confounding factors on diagnostic accuracy as well as the attempts made with respect to the improvement in diagnosis?

Studies were ascertained through a systematic search including four electronic databases (SCOPUS, PubMed, Google Scholar, Google search) available until March 15, 2020. We considered studies in wild mammals in which TB has been reported and we collected data using key elements. Search terms and key elements were combined with the Boolean operators (AND, OR, NOT), resulting in search algorithms which are shown in Additional file 1. The reports obtained for this systematic review were subjected to three screening phases as shown in Figure 1.

\section{Results and discussion}

A total of 336 articles were retrieved through search engines (196 by Scopus, 87 by Google scholar, 42 by Pubmed and 11 by Google search). Seventy-four articles were removed in first screening and 47 articles were eliminated in second screening. Finally, 124 articles were considered for systematic review after third screening (Figure 1). Different diagnostic tests include detection of TB like 


\section{Diagnosis of wildlife TB}

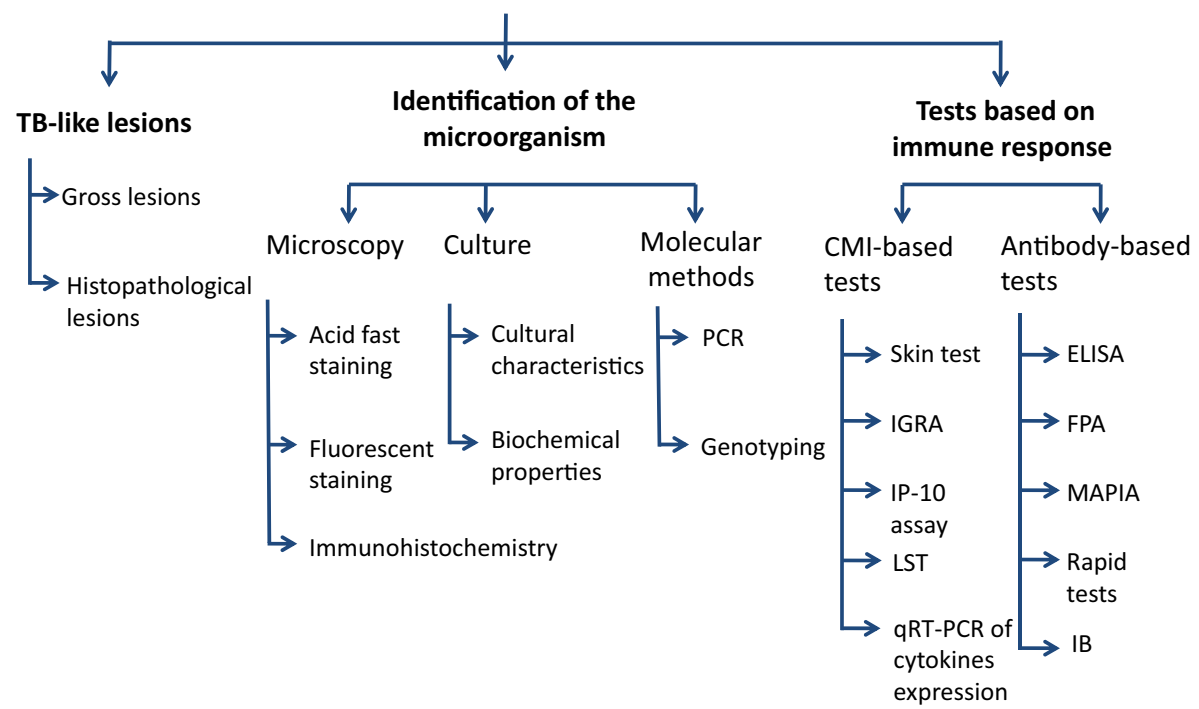

Figure 2 Principal methods for diagnosis of wildlife tuberculosis. TB: tuberculosis; CMI: cell-mediated immunity; PCR: polymerase chain reaction; IGRA: interferongamma release assay; IP-10 assay: interferon gamma-inducible protein 10; LST: lymphocyte stimulation test; qRT-PCR:quantitative reverse-transcription polymerase chain reaction assay; ELISA: enzyme-linked immunosorbent assay; FPA:fluorescence polarization assay; MAPIA: multiantigen print immunoassay; IB: immunoblotting.

lesions (TBL) (macroscopic or microscopic), identification of the microorganism (microscopy/culture and isolation/molecular methods) and immunological methods (cell-mediated immune (CMI) and antibody-based tests). Different tests used for TB diagnosis are schematically represented in Figure 2 . The tests can also easily be divided into two groups: tests detecting the pathogen (culture, molecular methods and microscopy) or tests focusing on host response (necropsy, histopathology and immunological methods). Major confounding factors are host, environment, habitat, management factors, prior sensitization, history of vaccination and other infections, sampling factors and technical factors related to diagnosis. The strategies for improving diagnosis comprise selection of appropriate test, proper implementation and interpretation of the test as well as combination of different tests for interpreting the results.

\section{Principal methods of TB diagnosis}

Ideally, a diagnostic test should need a small sample volume with simplicity in its collection and storage before performing the tests and it should be easy to carry out and interpret. It should also be relatively inexpensive, and with high Se and specificity (Sp). An overview of all diagnostic tests is shown in Additional file 2.

\section{TB like lesions (TBL)}

Presence of TBL is indicative of TB infection and is observed either macroscopically during post-mortem examination or microscopically during histopathological examination.

Post-mortem examination Post-mortem examination is the primary, sensitive and cost-effective method of disease surveillance [32]. This examination can be performed on hunted/dead animals by macroscopic examination of lymph nodes (LNs), and thoracic and abdominal organs, especially lungs to assess the TBL [33]. TBL includes nodular off-white lesions containing caseous material, which may be mineralized in the center and encapsulated by fibrous tissue $[23,34]$. The type, severity and place of the lesions can vary depending on the host, route of infection, stage of the disease as well as host-pathogen interaction $[35,36]$. Hence, the severity of lesions is negatively associated with protection against disease, which is helpful in the studies related to the efficacy of vaccine experiments [35]. In general, the gross pathological lesions are "noticeable" towards the advanced stages of the disease [23]. However, MTC infection can cause "latent" non-visible lesions in many wild animals, which result in a difficult diagnosis by post-mortem $[31,37]$. In Eurasian badger, the majority of infections are latent and hence gross patho- 
logical examination provides limited diagnostic Se in this species [34]. Moreover, lesions sometimes are limited to non-classical sites like prescapular or popliteal LNs as a result of MTC transmission by bite wound [38]. The gross TBL cannot be used as a confirmatory TB diagnosis because other non-tuberculous mycobacteria and pathogens other than mycobacteria (i.e. Corynebacterium pseudotuberculosis) may cause indistinguishable macroscopic lesions [39]. Hence, post-mortem findings in combination with other diagnostic techniques would be more effective in detecting the disease [40].

Histopathological examination The histological diagnosis involves pathological observation of the "classical tuberculous granuloma", which consists of a circular lesion formed by cells of inflammatory nature such as macrophages, epithelioid cells, lymphocytes and in some species, Langhans multinucleated giant cells surrounding a central region of developing necrosis ("caseating tubercles"), although non-necrotizing granulomatous encapsulation may also be present [34, 37, 41]. Granulomatous encapsulation with a fibrotic ring and mineralized necrosis in the center is considered as a key factor for containing TB infection [42]. Histopathological examination is not $100 \%$ specific for detecting MTC infection due to the detection of similar lesions in other non-tuberculous mycobacteria infections, therefore it requires complementary techniques for confirmation [43]. Despite of that it has many advantages; among them are that focal/ latent lesions can be detected as well as different histopathological studies have helped to discern target organs for sampling, i.e. tonsils and submandibular LNs in wild boar, mesenteric LNs in red deer or hepatic LNs in badgers [34, 37].

\section{Identification of the microorganism}

Identification of MTC organisms is a confirmatory method in $\mathrm{TB}$ diagnosis that can be performed by direct microscopy, culture or molecular methods for identification.

Microscopy Ziehl-Neelsen (ZN) staining is used to identify acid-fast bacteria, mainly mycobacteria, and can increase the reliability of TB diagnosis [44]. This is a simple, rapid and economical method for the detection of mycobacteria, but it is not $100 \%$ specific due to the detection of other non-tuberculous mycobacteria [42]. Samples can be collected from a slaughtered/hunted animal or a live animal (samples of tracheobronchial washes). ZN staining is commonly used in histological sections which improves the diagnostic Se.

Immunofluorescence and immunohistochemistry include the staining of mycobacteria using monoclonal or polyclonal antibodies. These techniques provide more accurate results than acid fast staining [43], but they are laborious and less economical, along with the fact that sometimes polyclonal antibodies can give non-specific results due to cross-reactions with other non-tuberculous mycobacteria [45].

Microbiological culture Culture of microorganism and identification are considered as the gold standard method for the diagnosis of TB in all wild animals [46]. It is employed as a post-mortem diagnostic method by collecting the samples especially from organs with TBL $[19,36]$ or pooled LN samples in case of non-visible TBL [28]. Culture from bronchoalveolar lavage (BAL)/tracheal washing can be used for ante-mortem diagnosis in some wild animals like lion [13], badger [43] and wild meerkat [9]. However, Se can be variable due to the lack of active shedding of the microorganism from infected animals and hence the absence of mycobacteria in the collected sample [47]. In addition, the decontamination step prior to MTC culture can adversely affect the viability of mycobacteria, especially when the number of viable microorganisms is low, and it may, therefore, lead to false negative results in the culture [24]. In elephants, trunk wash culture is the officially recommended ante-mortem diagnostic test in USA [10], but the contamination by other pathogens is a major problem [48]. The Se of culture and isolation varies depending on the stage of the disease (latent or noticeable), the number and selection of tissues processed and on the sample quality [49]. Culture is expensive, time-consuming and requires biosafety level 3 laboratories [49]. However, live sampling is highly relevant, as it is important to detect the excretion of pathogens and thereby determine the possibility of transmission (continuous studies are possible with re-sampling). It is also important for determining the infection rate before and after applying control methods like vaccination as well as to detect the safety of vaccine $[19,30]$. Identification of the microorganism after cultural isolation can be done by colony characteristics, biochemical tests or nucleic acid recognition methods [50]. Moreover, culture is performed on specific medium which supports the confirmation that the bacteria is from the MTC.

Molecular identification Nucleic acid recognition methods can be applied using DNA extracted directly from tissue or clinical samples (not blood) or from the growing colonies. DNA extraction from growing colonies is more efficient since growing colonies usually contain higher bacterial load (approximately more than 50 colony forming units (CFU)/g) and are also usually less contaminated than tissues or clinical samples, which facilitates the extraction. The diagnosis of MTC by direct polymerase 
chain reaction (PCR) is fast and highly sensitive, showing great value in epidemiological studies [51] and, it is highly useful to reach immediate treatment decisions in some species [48]. The test has variable Se due to absence of organism in collected sample, which in turn depends on many factors like stage of disease, intensity of infection etc. Sample quality is a major factor regarding test result because presence of DNAases can degrade DNA resulting in false negative result. Moreover PCR requires costly reagents and equipment $[48,51]$.

Another nucleic acid recognition method is genotyping, which comprises spoligotyping, deep sequencing, Restriction Fragment Length Polymorphism (RFLP) and Mycobacterial Interspersed Repetitive Units-Variable Number of Tandem Repeats (MIRU-VNTR) analysis [52]. Genotyping can also be used to discriminate different members and strains of MTC species, to elucidate transmission patterns, to perform large-scale molecular epidemiological studies [53] or to detect outbreaks and their sources by MIRU-VNTR [54].

\section{Tests based on immune response}

The immunological TB diagnosis is based on CMI and humoral mediated diagnostic tests using different antigens. The main antigen used in diagnostics is bovine tuberculin or bovine purified protein derivative (bPPD), which is a combination of proteins extracted from $M$. bovis. Another antigen used in diagnostics is lipoarabinomannan (LAM), which is a glycolipid as well as a virulence factor associated with MTC [55]. The specific antigens used in TB diagnosis include proteins from $M$. bovis MPB83 and MPB70, which are homologous proteins within MTC members, but have difference in electrophoretic mobility and isoelectric point [56], early secretory antigenic target- $6 \mathrm{kDa}$ (ESAT-6) and culture filtrate protein-10 $\mathrm{kDa}$ (CFP-10), which are virulence proteins of MTC absent in most of the non-tuberculous mycobacteria and $M$. bovis Bacillus Calmette Guerin (BCG) [57], and cell wall proteins like Rv3615c and Rv3020c, absent in most of the non-tuberculous mycobacteria and M. bovis BCG [58]. The antigen (Ag) 85A is a secretory protein of $M$. tuberculosis and BCG, but it does not have much relevance in diagnosis of animal TB [20]. The P22 antigen is a recently immunopurified protein complex from bPPD comprising mainly the proteins MPB70, MPB83, ESAT-6 and CFP-10 [59]. The details in immunological techniques used for TB diagnosis of wildlife are listed in Tables 1, 2, 3, 4, 5, 6, 7 and 8.

CMI based diagnostics Cell mediated immunity based diagnostic tests are based on the type IV or delayed hypersensitivity in which sensitized $\mathrm{T}$ cells produce cytokines, mainly interferon gamma (IFNY), interleukin-2 (IL-2) and
IL-16, as well as chemokines resulting in mast cell degranulation and production of histamine [60]. They are mainly used to detect initial stages of the disease.

\section{Skin test}

Skin test is an ante-mortem TB diagnostic test that is not usually applicable to wildlife due to the need to handle animals twice over a 2-3 day interval [61]; however it can be used in some captive wildlife and zoo animals. Generally, the test involves the intradermal inoculation of tuberculous antigen (mostly bPPD) and measuring the skin thickness after $72 \mathrm{~h}$, which is called single intradermal tuberculin skin test (SIT). Another type of skin test is single comparative intradermal tuberculin skin test (SCITT) in which avian purified protein derivative (aPPD) is also used in addition to bPPD in order to avoid non-specific reaction of Mycobacterium avium complex (MAC) [22]. The optimum time for second reading of skin test is $72 \mathrm{~h}$ [21]. Skin testing is relatively less expensive, but it does not support differentiation of infected and vaccinated animals (DIVA) testing. Skin test has been tested in different wild animal species with varying level of diagnostic accuracy. Sites of inoculation also differ depending on the species assayed, like neck region in deer [21], lion [62], nyala [12] and African buffalo [63]; chest wall over the posterior rib cage in badger [64]; caudal fold (caudal fold test-CFT) in elephant [65] and African buffalo [66]; inguinal region in wild boar [22]; or caudal part of the ear in warthog and pygmy hippopotamus $[8,67]$. In cattle, the interpretation of SIT is based on the scoring of reactions higher than $4 \mathrm{~mm}$ as standard reactor, higher than $2 \mathrm{~mm}$ and less than $4 \mathrm{~mm}$ as inconclusive reactor and less than $2 \mathrm{~mm}$ as negative reactor. In SCITT, any animal with a skin fold increase greater than $2 \mathrm{~mm}$ to bPPD and bPPD > aPPD (at least $1 \mathrm{~mm}$ ) is considered as TB reactor $[13,68]$.

This method is the official ante-mortem test in farmed deer as approved by Office international des epizooties (OIE) [69]. In wild bovid species like African buffalo or Kafue lechwe, skin test is routinely used for diagnosis of TB as well as in test and slaughter programs $[70,71]$. Even so, in many other species, this technique is less reliable or has less diagnostic accuracy or rather limited information is available. Black skin of many species of swine restricts the implementation of skin test [72]. The test is not practical in pachyderm animals like elephant and rhinoceros [73]. In possum and badger, the test is not reliable due to the weak responses produced [74]. Generally, tuberculin skin test has some drawbacks including the need to handle the animals twice in a 72-h period and stress associated with this double handling which can influence the results [75], technical variability [76], low Sp [77] and reduced Se towards later stages of the disease 
Table 1 Details of immunological diagnosis in Cervidae.

\begin{tabular}{|c|c|c|c|c|c|c|c|}
\hline Assay test & Species & $N / E$ & $n_{s e}+n_{s p}$ & Antigens & Se (\%) & Sp (\%) & References \\
\hline \multirow[t]{6}{*}{ Skin test (SCITT) } & Red deer & $N+E$ & $60+1157$ & bPPD, aPPD & 91.4 & 98.7 & [144] \\
\hline & Red deer & N & 218 & bPPD, aPPD & $\mathrm{NE}$ & 46.9 & [126] \\
\hline & Elk & $N$ & $7+3$ & bPPD, aPPD & 100 & 100 & [145] \\
\hline & Reindeer & $E$ & $13+4$ & bPPD, aPPD & 92 & 25 & [146] \\
\hline & White tailed deer & $N+E$ & $60+56$ & bPPD, aPPD & 97 & 81 & [61] \\
\hline & Fallow deer & N & 21 & bPPD, aPPD & 80.1 & $N E$ & [21] \\
\hline \multirow[t]{12}{*}{ IGRA } & White tailed deer & $E$ & $91+44$ & bPPD, aPPD & 74 & 98 & [83] \\
\hline & Elk & $N$ & 51 & bPPD, aPPD & $N E$ & $90^{\mathrm{a}}, 78^{\mathrm{b}}$ & [79] \\
\hline & & & & ESAT-6/CFP-10 & $N E$ & $\begin{array}{l}100^{c} \\
96^{d}\end{array}$ & \\
\hline & Reindeer & $\mathrm{N}$ & 106 & bPPD, aPPD & $\mathrm{NE}$ & $91^{a} 83^{b}$ & \\
\hline & & & & ESAT-6/CFP-10 & $\mathrm{NE}$ & $94^{c}, 87.5^{d}$ & \\
\hline & White tailed deer & $N$ & 95 & bPPD & $\mathrm{NE}$ & $98^{a}, 92^{b}$ & \\
\hline & & & & ESAT-6/CFP-10 & $\mathrm{NE}$ & $97^{c}, 95^{d}$ & \\
\hline & Red deer & $E$ & 15 & bPPD & $92.8^{e}, 100^{f}, 75^{g}$ & 100 & [23] \\
\hline & & & & P22 & $92.8^{e}, 100^{f}, 87.5^{g}$ & 100 & \\
\hline & & & & ESAT-6/CFP-10 & $92.3^{e}, 100^{f}, 75^{g}$ & 100 & \\
\hline & & & & Rv3615c & $40^{\mathrm{e}}, 26.6^{\mathrm{f}}, 37.5^{\mathrm{g}}$ & 100 & \\
\hline & & & & Rv3020c & $66.6^{\mathrm{e}}, 100^{\mathrm{f}}, 87.5^{\mathrm{g}}$ & 100 & \\
\hline \multirow[t]{5}{*}{ LST } & Elk & $N$ & $66+324$ & bPPD & 70 & 74 & [147] \\
\hline & Elk & $N$ & $33+450$ & bPPD & 83 & 64 & [104] \\
\hline & Red deer & N & $39+16$ & bPPD & 95 & 44 & [68] \\
\hline & & & & MPB70 & 72 & 50 & \\
\hline & Red deer-elk hybrid & $E$ & $10+15$ & bPPD & 65.7 & 92.5 & [84] \\
\hline $\begin{array}{l}\text { qRT-PCR of cytokines } \\
\text { expression }\end{array}$ & Red deer-elk hybrid & $E$ & $10+15$ & bPPD & 78.6 & 97.5 & [84] \\
\hline \multirow[t]{8}{*}{ ELISA } & Red deer & $N$ & $104+56$ & $\begin{array}{l}\text { bPPD } \\
\text { MPB70 }\end{array}$ & $\begin{array}{l}88 \\
80\end{array}$ & $\begin{array}{l}52 \\
79\end{array}$ & [68] \\
\hline & Red deer & N & $94+217$ & bPPD, MPB70, aPPD & 45.7 & 100 & [126] \\
\hline & Red deer & $E$ & $15+15$ & Ethanol Extract of M. bovis & 86.7 & 93.3 & [102] \\
\hline & Red deer & N & $221+204$ & $\begin{array}{l}\text { bPPD } \\
\text { P22 }\end{array}$ & $\begin{array}{l}70.1 \\
70.1\end{array}$ & $\begin{array}{l}91.6 \\
99\end{array}$ & [24] \\
\hline & Elk & $N$ & $108+48$ & MPB83 & 49.1 & 97.9 & [138] \\
\hline & Fallowdeer & $N$ & $73+157$ & bPPD & 51 & 96 & [120] \\
\hline & White tailed deer & N & $12+329$ & LAM enriched antigen & $66.7^{h}, 58.3^{i}$ & $\begin{array}{l}95.1^{\mathrm{h}} \\
97.3^{\mathrm{i}}\end{array}$ & [113] \\
\hline & Reindeer & $E$ & $11+4$ & LAM & 100 & 50 & [55] \\
\hline BTB & Red deer & $N$ & $87+200$ & bPPD, aPPD & 90.8 & 98 & [126] \\
\hline \multirow[t]{3}{*}{ FPA } & Red deer and elk & $\mathrm{N}$ & 16 & MPB70 & 81 & $\mathrm{NE}$ & [91] \\
\hline & Elk & $N$ & $33+450$ & MPB70 & 40 & 81 & [104] \\
\hline & Red deer-elk hybrid & $E$ & 10 & MPB70 & 33.33 & $\mathrm{NE}$ & {$[75]$} \\
\hline RT & White tailed deer & $\mathrm{N} / \mathrm{E}$ & $28+435$ & ESAT-6, CFP-10, MPB83 & 75 & 98.9 & [109] \\
\hline \multirow[t]{8}{*}{ Cervid TB STAT-PAK } & Elk & $N$ & $31+842$ & ESAT-6, CFP-10, MPB83 & 87.1 & 98.3 & [148] \\
\hline & Elk & $N$ & $33+450$ & ESAT-6, CFP-10, MPB83 & 62 & 87 & [104] \\
\hline & $\begin{array}{l}\text { Elk } \\
\text { Fallow deer }\end{array}$ & $N$ & $\begin{array}{l}34+141 \\
32+107\end{array}$ & ESAT-6, CFP-10, MPB83 & $\begin{array}{l}82 \\
91\end{array}$ & $\begin{array}{l}93 \\
91\end{array}$ & [107] \\
\hline & Fallow deer & $N$ & 21 & ESAT-6, CFP-10, MPB83 & 80.1 & $\mathrm{NE}$ & [21] \\
\hline & Red deer-elk hybrid & $E$ & 10 & ESAT-6, CFP-10, MPB83 & 72.5 & NE & [75] \\
\hline & White tailed deer & N & $22+724$ & ESAT-6, CFP-10, MPB83 & 54.5 & 98.1 & [113] \\
\hline & Red deer & $\mathrm{N} / \mathrm{E}$ & $52+105$ & ESAT-6, CFP-10, MPB83 & 86.5 & 83.8 & [119] \\
\hline & Mixed Deer sp. & N & $7+425$ & ESAT-6, CFP-10, MPB83 & $85.7^{j}$ & $94.8^{j}$ & [119] \\
\hline
\end{tabular}


Table 1 (continued)

\begin{tabular}{|c|c|c|c|c|c|c|c|}
\hline Assay test & Species & N/E & $n_{s e}+n_{s p}$ & Antigens & Se (\%) & $\mathrm{Sp}(\%)$ & References \\
\hline \multirow[t]{4}{*}{$\begin{array}{l}\text { DPP } \\
\text { Vet TB }\end{array}$} & $\begin{array}{l}\text { Elk } \\
\text { Fallow deer }\end{array}$ & $\mathrm{N}$ & $\begin{array}{l}34+141 \\
32+107\end{array}$ & ESAT-6, CFP-10, MPB83 & $\begin{array}{l}79 \\
91\end{array}$ & $\begin{array}{l}98 \\
99\end{array}$ & [107] \\
\hline & Fallow deer & $\mathrm{N}$ & $73+157$ & ESAT-6, CFP-10, MPB83 & 71 & 88 & [120] \\
\hline & Red deer & N/E & $52+105$ & ESAT-6, CFP-10, MPB83 & 84.6 & 91.4 & [119] \\
\hline & White tailed deer & N/E & $63+903$ & ESAT-6, CFP-10, MPB83 & 65.1 & 97.8 & [121] \\
\hline \multirow[t]{4}{*}{ MAPIA } & $\begin{array}{l}\text { Elk } \\
\text { Fallow deer }\end{array}$ & N & $\begin{array}{l}34 \\
32\end{array}$ & M. bovis antigens ${ }^{k}$ & $\begin{array}{l}82 \\
97\end{array}$ & $\begin{array}{l}\mathrm{NE} \\
\mathrm{NE}\end{array}$ & [107] \\
\hline & Red deer-elk hybrid & E & 10 & M. bovis antigens ${ }^{k}$ & 76.7 & $\mathrm{NE}$ & {$[75]$} \\
\hline & White tailed deer & N & $22+727$ & M. bovis antigens ${ }^{k}$ & 68.2 & 97.1 & [113] \\
\hline & Reindeer & $\mathrm{E}$ & $11+23$ & M. bovis antigens ${ }^{k}$ & 100 & 85 & {$[55]$} \\
\hline \multirow[t]{2}{*}{ IB } & White tailed deer & $\mathrm{N}$ & $\begin{array}{l}20+671 \\
13+333\end{array}$ & $\begin{array}{l}\text { MPB83 } \\
\text { Whole cell sonicate }\end{array}$ & $\begin{array}{l}55 \\
46.2\end{array}$ & $\begin{array}{l}99.3 \\
92.5\end{array}$ & [113] \\
\hline & Reindeer & E & $11+4$ & Whole cell sonicate & 90.9 & 50 & {$[55]$} \\
\hline
\end{tabular}

N/E: natural or experimental infection; $\mathrm{n}_{\mathrm{Se}}$ : number of TB positive animals used for evaluation of Se; $\mathrm{n}_{\mathrm{sp}}$ : number of TB negative animals used for evaluation of Sp; Se: sensitivity; Sp: specificity; SCITT: single comparative intradermal tuberculin skin test; LST: lymphocyte stimulation test; qRT-PCR: quantitative reverse-transcription polymerase chain reaction assay; IGRA: interferon gamma release assay; ELISA: enzyme linked immunosorbent assay; BTB: blood tuberculosis test; FPA: fluorescent polarization assay; MAPIA: multiantigenimmunoprint assay; Cervid TB STAT-PAK and DPP Vet TB: lateral flow tests (Chembio Diagnostic Systems, Inc., USA); RT: lateral flow rapid test (name not mentioned-Chembio Diagnostic Systems, Inc., USA); NE: not estimated; IB: immunoblotting

a Cut off: OD of bPPD-aPPD and bPPD-PBS $>0.1$.

b Cut off: OD of bPPD-aPPD and bPPD-PBS $>0.05$

c Cut off: OD of ESAT-6:CFP-10-PBS $>0.1$.

d Cut off: OD of ESAT-6:CFP10-PBS $>0.05$.

e Se of experimentally infected animals at 15 days post-infection (dpi).

$f$ Se of experimentally infected animals at $30 \mathrm{dpi}$.

9 Se of experimentally infected animals at $60 \mathrm{dpi}$ (cut off for e, $\mathrm{f}$, g: for bPPD and P22, OD of bPPD-aPPD and bPPD-PBS >0.05, for specific antigens like ESAT-6:CFP10, Rv3615c and Rv3020c, OD of specific antigen-PBS $>0.05$ ).

h Cut off: $O D \geq 0.25$.

i Cut off: $O D \geq 0.3$.

j Se and Sp were evaluated based on mixed species of deer consisting of fallow deer, roe deer and red deer.

k ESAT-6, CFP-10, MPB59, MPB64, MPB70, MPB83, 16-kDa protein, 38-kDa protein, CFP-10/ESAT-6 and 16 kDa protein/MPB83, and two native antigens, bovine PPD and $M$. bovis culture filtrate.

[78]. Moreover, the possibility of acute stress in tested animals could induce high levels of cortisol, resulting in reduced IFN $\gamma$ response $[66,79]$.

Whole blood interferon gamma release assay (IGRA)

The IGRA is used as an alternative or supplementary assay to the skin test. The test involves the measurement of CMI response in vitro by an assay that detects the IFN $\gamma$ produced by peripheral blood mononuclear cells (PBMCs) exposed to bPPD (or specific antigens like ESAT-6/CFP-10, P22, Rv3615c or Rv3020c) and aPPD antigens. The assay has been tested in deer [23], African buffalo [63], badger [80], elephant [73], white rhinoceros [11] and wild boar [36]. Briefly, the assay consists of two stages. First, heparinized whole blood is incubated with antigens (i.e., PPDs, specific MTC antigens along with positive controls as mitogens or superantigens (pokeweed mitogen, phytohemagluttinin or staphylococcal enterotoxin B) and negative controls as phosphate buffered saline (PBS) or Roswell Park Memorial Institute (RPMI) Medium) for 18-24 h to induce production and release of IFN $\gamma$ predominantly by $\mathrm{T}$ lymphocytes. Secondly, IFNy present in the plasma supernatants is quantified in a sandwich ELISA using two species specific antibodies against IFN $\gamma$ (capture and detection antibody) $[23,63,80]$. Usually, the difference in the optical density (OD) of antigens with negative control and aPPD is taken into consideration for interpreting the results. The interpretation of the test varies with many factors like species, type of antigen/antibody used, preferred $\mathrm{Se} / \mathrm{Sp}$, as well as purpose of the test $[23,81]$.

IGRA has many practical advantages over the skin test in wildlife, as the test avoids the stimulation of live animal with mycobacterial antigens, as well as it needs only a single sample collection and less technical variability compared to skin test $[23,81]$. Moreover, IGRA can be applied sequentially to get a conclusive result if there is a doubt or inconclusive result in the skin test, as well as 
Table 2 Details of immunological diagnosis in wild suids.

\begin{tabular}{|c|c|c|c|c|c|c|c|}
\hline Assay test & Species & N/E & $\mathrm{n}_{\mathrm{se}}+\mathrm{n}_{\mathrm{sp}}$ & Antigen & Se (\%) & $\mathrm{Sp}(\%)$ & References \\
\hline \multirow[t]{2}{*}{ Skin test (SCITT) } & Eurasian wild boar & $E$ & $4+31$ & bPPD, aPPD & $77-100^{\mathrm{a}}$ & $48.4-77.4^{\mathrm{a}}$ & [22] \\
\hline & Common warthog & $\mathrm{N}$ & $16+18$ & bPPD, aPPD & 81 & 100 & [8] \\
\hline Skin test (SIT) & Common warthog & $\mathrm{N}$ & $16+18$ & bPPD, aPPD & 69 & 100 & \\
\hline ELISA- protein G & Eurasian wild boar & $N$ & $96+104$ & bPPD & 79.2 & 100 & [133] \\
\hline \multirow[t]{2}{*}{ ELISA- IgG } & Eurasian wild boar, domestic pig & N & $277+366$ & $\begin{array}{l}\text { bPPD } \\
\text { P22 }\end{array}$ & $\begin{array}{l}77.3 \\
84.1\end{array}$ & $\begin{array}{l}97.3 \\
98.4\end{array}$ & [25] \\
\hline & Eurasian wild boar & $\begin{array}{l}N \\
E\end{array}$ & $\begin{array}{l}30+25 \\
51+9\end{array}$ & $\begin{array}{l}\text { P22 } \\
\text { P22 }\end{array}$ & $\begin{array}{l}96.7 \\
84.3\end{array}$ & $\begin{array}{l}100 \\
100\end{array}$ & [125] \\
\hline \multirow[t]{3}{*}{ ELISA- protein A\&G } & Eurasian wild boar & $\mathrm{N}$ & $22+43$ & MPB83 & 86 & 100 & [149] \\
\hline & Common warthog & $\mathrm{N}$ & $16+19$ & bPPD & 88 & 89 & [97] \\
\hline & Common warthog & N & 25 & bPPD & 92 & NE & [150] \\
\hline \multirow[t]{3}{*}{ ELISA TB-VK } & Eurasian wild boar & $\mathrm{N}$ & $73+112$ & bPPD & 72.6 & 96.4 & [137] \\
\hline & Common warthog & $\mathrm{N}$ & $16+19$ & bPPD & 88 & 79 & [97] \\
\hline & Common warthog & $\mathrm{N}$ & 25 & bPPD & 86 & $N E$ & [150] \\
\hline ELISA-INgezim TB porcine & Eurasian wild boar & $\begin{array}{l}N \\
E\end{array}$ & $\begin{array}{l}30+25 \\
51+9\end{array}$ & MPB70, MPB83 & $\begin{array}{l}100 \\
92.1\end{array}$ & $\begin{array}{l}100 \\
100\end{array}$ & [126] \\
\hline $\begin{array}{l}\text { INgezim } \\
\text { Tuberculosis DR }\end{array}$ & Eurasian wild boar & $\begin{array}{l}N \\
E\end{array}$ & $\begin{array}{l}30+25 \\
51+9\end{array}$ & MPB83 & $\begin{array}{l}93.3 \\
86.2\end{array}$ & $\begin{array}{l}100 \\
100\end{array}$ & [125] \\
\hline RT & Eurasian wild boar & N & $64+113$ & ESAT-6, CFP-10, MPB83 & 76.6 & 97.3 & [109] \\
\hline \multirow[t]{3}{*}{ DPP VetTB } & Warthog Eurasian wild boar & $\mathrm{N}$ & $\begin{array}{l}23+35 \\
56+30\end{array}$ & ESAT-6, CFP-10, MPB83 & $\begin{array}{l}82.6 \\
80.4\end{array}$ & $\begin{array}{l}91.4 \\
96.7\end{array}$ & [110] \\
\hline & Eurasian wild boar & $N$ & $96+104$ & ESAT-6, CFP-10, MPB83 & 89.6 & 90.4 & [133] \\
\hline & Common warthog & $\mathrm{N}$ & $16+19$ & ESAT-6, CFP-10, MPB83 & 75 & 89 & [97] \\
\hline INgezim TB-CROM & Eurasian wild boar & $\begin{array}{l}N \\
E\end{array}$ & $\begin{array}{l}30+25 \\
51+9\end{array}$ & MPB83 & $\begin{array}{l}93.3 \\
90.2\end{array}$ & $\begin{array}{l}96 \\
100\end{array}$ & [125] \\
\hline
\end{tabular}

N/E: natural or experimental infection; Se: sensitivity; Sp: specificity; $\mathrm{n}_{\mathrm{Se}}$ : number of TB positive animals used for evaluation of Se; $\mathrm{n}_{\mathrm{Sp}}$ : number of TB negative animals used for evaluation of $\mathrm{Sp} ;{ }^{a}$ range of values due to the difference in reading criteria; SIT: single intradermal tuberculin test; SCITT: single comparative intradermal tuberculin skin test; IGRA: interferon gamma assay; ELISA: enzyme linked immunosorbent assay; ELISA TB-VK: Commercial ELISA kit (Vacunek, Spain); ELISA- INgezim TB porcine and INgezim Tuberculosis DR: Commercial ELISA kit (INGENASA, Spain); RT: lateral flow rapid test (name not mentioned-Chembio Diagnostic Systems, Inc., USA); DPP VetTB: rapid lateral flow kit (Chembio Diagnostic Systems, Inc., USA), INgezim TB-CROM: rapid lateral flow kit (INGENASA S.L., Spain).

in association with other antibody mediated diagnostic measures to improve the Se and Sp [23]. However, the test involves logistical constraints such as strict laboratory conditions and the need for fast processing of samples [82].

In wild bovids, like African buffalo and nyala, the commercial IGRA test usually used is Bovigam assay with bPPD (Prionics, Zurich, Switzerland) [12]. Cervigam assay (Pfizer Animal Health, New York, USA) was the commercial IGRA test developed for use in deer [83, 84]. However, Cervigam assay is not commercially available nowadays, since a lack of adequate response to mycobacterial antigens has been reported in infected white-tailed deer, elk and fallow deer [79]. Offering the greatest potential for the improvement of $\mathrm{Sp}$, the use of specific antigens like ESAT-6, CFP-10, P22, TB-7, Rv3615c or Rv3020c in IGRA has been carried out in many wild animal species like African buffalo [63], badger [80] and red deer [23]. In African buffalo, the commercially available Bovigam PC-EC assay, based on
ESAT-6:CFP-10 proteins, and Bovigam PC-HP assay, based on ESAT-6/CFP-10 proteins, peptides simulating Rv3615c and three additional mycobacterial antigens, are promising diagnostic approaches [85-87]. In red deer, IGRA with antigens bPPD, P22 and the combination of ESAT-6/CFP-10 and Rv3020c was able to detect the infection as early as 15 days post-experimental challenge [23]. Moreover, IGRA based on ESAT-6/CFP-10 is able to differentiate between BCG vaccinated and infected animals $[20,23,36]$. On the other hand, the use of specific or purified antigens can result in lack of suitable Se in badger [80]. Nevertheless, there are some strategies focusing on new antigen stimulating platforms in order to counteract this loss of Se. Thus, modified QuantiFERON ${ }^{\circledR}$ TB Gold In-Tube (mQFT) system is an IGRA which utilizes peptides simulating ESAT-6 and CFP-10 antigens, as well as TB7 in three blood collection tubes for the stimulation of PBMCs to produce IFN $\gamma$, and it provided better Sp without losing Se in 
Table 3 Details of immunological diagnosis in European badger.

\begin{tabular}{|c|c|c|c|c|c|c|}
\hline Assay test & N/E & $\mathrm{n}_{\mathrm{Se}+} \mathrm{n}_{\mathrm{Sp}}$ & Antigen & Se (\%) & Sp (\%) & References \\
\hline Skin test & $\mathrm{N}$ & $10+37$ & $\begin{array}{l}\text { bPPD, NT, whole killed bovine } \\
\text { tubercle bacilli }\end{array}$ & 70 & 73 & [64] \\
\hline \multirow[t]{6}{*}{ IGRA } & \multirow[t]{2}{*}{$\mathrm{N}$} & \multirow[t]{2}{*}{$46+185$} & bPPD, aPPD & $\begin{array}{l}74.5 \\
80.9^{\mathrm{a}}\end{array}$ & $\begin{array}{l}93.6 \\
93.6^{\mathrm{a}}\end{array}$ & \multirow[t]{2}{*}{ [80] } \\
\hline & & & ESAT-6/CFP-10 & $\begin{array}{l}60.9 \\
60.9^{a}\end{array}$ & $\begin{array}{l}94.6 \\
94.6^{\text {a }}\end{array}$ & \\
\hline & $\mathrm{N}$ & ND & bPPD, aPPD & 79.9 & 95 & [143] \\
\hline & \multirow[t]{2}{*}{ N } & $39+147$ & \multirow[t]{2}{*}{ bPPD, aPPD } & $84.6^{b}$ & $92.5^{b}$ & \multirow[t]{2}{*}{ [96] } \\
\hline & & $7+40$ & & $57.1^{c}$ & $97.5^{c}$ & \\
\hline & N & ND & bPPD, aPPD & 52 & 97 & {$[142]$} \\
\hline LTA & $\mathrm{N}$ & $8+13$ & bPPD, aPPD & 87.5 & 84.6 & [92] \\
\hline $\begin{array}{l}\text { qRT-PCR of cytokines } \\
\text { expression }\end{array}$ & $\mathrm{N}$ & $53+194$ & bPPD, ESAT-6/CFP-10 & 64.2 & 93.3 & [93] \\
\hline \multirow[t]{2}{*}{ ELISA } & $\mathrm{N}$ & $25+28$ & \multirow[t]{2}{*}{ P22 } & $80^{d}, 76^{e}$ & $85.7^{d}, 85.7^{e}$ & \multirow[t]{2}{*}{ [99] } \\
\hline & E & $34+36$ & & $82^{d}, 79^{e}$ & $80.56^{\mathrm{d}}, 83.3^{\mathrm{e}}$ & \\
\hline \multirow[t]{6}{*}{ Brock test } & N & $8+13$ & MPB83 & 62.5 & 100 & [92] \\
\hline & $\mathrm{N}$ & $41+33$ & MPB83 & 46 & 82 & [95] \\
\hline & \multirow[t]{2}{*}{ N } & $340+817$ & \multirow[t]{2}{*}{ MPB83 } & $54.7^{b}$ & $93^{\mathrm{b}}$ & \multirow[t]{2}{*}{ [96] } \\
\hline & & $39+238$ & & $53.9^{c}$ & $96.6^{c}$ & \\
\hline & $\mathrm{N}$ & $51+193$ & MPB83 & 52.9 & 90.7 & {$[93]$} \\
\hline & $\mathrm{N}$ & $78+100$ & MPB83 & 47.4 & 89 & [101] \\
\hline Dachs TB ELISA & $\mathrm{N}$ & $41+33$ & MPB83 & 61 & 82 & {$[95]$} \\
\hline MAPIA & N & $78+100$ & $\begin{array}{l}\text { M. bovis antigens }{ }^{f} \\
\text { M. bovis antigens }{ }^{g}\end{array}$ & $\begin{array}{l}48.7 \\
59\end{array}$ & $\begin{array}{l}88 \\
84\end{array}$ & [101] \\
\hline \multirow[t]{4}{*}{ BrockTB STAT-PAK } & $\mathrm{N}$ & $\begin{array}{l}340+817 \\
39+238\end{array}$ & ESAT-6, CFP-10, MPB83 & $\begin{array}{l}49.7^{b} \\
56.4^{c}\end{array}$ & $\begin{array}{l}92.5^{b} \\
96.2^{c}\end{array}$ & [96] \\
\hline & $\mathrm{N}$ & ND & ESAT-6, CFP-10, MPB83 & 50.4 & 96.9 & [143] \\
\hline & N & NM & ESAT-6, CFP-10, MPB83 & 59.09 & 66.67 & [139] \\
\hline & $\mathrm{N}$ & ND & ESAT-6, CFP-10, MPB83 & 58 & 97 & [142] \\
\hline DPP Vet TB & N & $38+418$ & ESAT-6, CFP-10, MPB83 & 50 & 95 & [123] \\
\hline \multirow[t]{2}{*}{ RT } & N & $454+1078$ & ESAT-6, CFP-10, MPB83 & 50.7 & 93.1 & [109] \\
\hline & $\mathrm{N}$ & $78+100$ & MPB83, TBF 10 & 52.6 & 95 & {$[101]$} \\
\hline
\end{tabular}

N/E: natural or experimental infection; $\mathrm{n}_{\mathrm{Se}}$ : number of TB positive animals used for evaluation of Se; $\mathrm{n}_{\mathrm{Sp}}$ : number of TB negative animals used for evaluation of Sp; Se: sensitivity; Sp: specificity; IGRA: interferon gamma release assay; ELISA: enzyme linked immunosorbent assay; LTA: comparative lymphocyte transformation assay; qRTPCR: quantitative reverse-transcription polymerase chain reaction assay; MAPIA: multiantigenimmunoprint assay; NT: new tuberculin; Brock TB STAT-PAK: lateral flow rapid test (Chembio Diagnostic Systems, Inc., USA); RT: rapid test (name not mentioned).

a Se and Sp based on rabbit monoclonal antiserum (mEIA) instead of polyclonal pair ( $p E I A$ ) of antibodies in other studies; ND: not defined, Se and Sp are calculated based on Bayesian analysis; NM: not mentioned in the article; NE: not estimated.

b Se or Sp in adults.

c Se or Sp in cubs.

d Indirect ELISA.

e Competitive ELISA.

f Recombinant antigens only i.e., ESAT-6, CFP-10,MPB59, MPB64, MPB70, MPB83, 16-kDa protein, 38-kDa protein, CFP-10/ESAT-6 and 16 kDa protein/MPB83.

$g$ Recombinant antigens and $M$. bovis culture filtrate.

African buffalo $[63,71,88]$, warthog [89] and white rhinoceros [11].

IGRA enzyme-linked immunospot assay (ELISPOT) A direct ELISPOT assay has also been conducted in some studies in badgers with a high diagnostic value $[20,52]$.
The assay is usually based on net bPPD response and the use of the other antigens only aims to increase Sp. The assay detects IFN $\gamma$ produced by PBMCs stimulated with bPPD and aPPD, CFP-10/ESAT-6, Ag85, P22 or mitogen Concanavalin A as positive control, all diluted in RPMI complete medium for 16-20 $\mathrm{h}$ in wells pre-coated with monoclonal antibody against IFN $\gamma$. The IFN $\gamma$ producing 
Table 4 Details of immunological diagnosis in wild bovids.

\begin{tabular}{|c|c|c|c|c|c|c|c|}
\hline Assay test & Species & N/E & $\mathrm{n}_{\mathrm{Se}}+\mathrm{n}_{\mathrm{Sp}}$ & Antigens & Se $(\%)$ & Sp (\%) & References \\
\hline \multirow[t]{2}{*}{ Skin test (CFT) } & Wild bison & N & ND & bPPD & 57.6 & 80.3 & {$[105]$} \\
\hline & Wild bison & N & $2+24$ & bPPD & 50 & 88 & {$[111]$} \\
\hline Skin test (SCITT) & African buffalo & $\mathrm{N}$ & 51 & bPPD, aPPD & 86.3 & $\mathrm{NE}$ & {$[88]$} \\
\hline \multirow[t]{7}{*}{ IGRA } & African buffalo & $N+E$ & $149+344$ & bPPD, aPPD & 92.1 & 68.3 & {$[81]$} \\
\hline & African buffalo & $\mathrm{N}$ & 8 & bPPD, aPPD & 100 & $\mathrm{NE}$ & {$[7]$} \\
\hline & & & & PC-HP, PC-EC & 75 & $\mathrm{NE}$ & \\
\hline & African buffalo & $N$ & 44 & ESAT-6, CFP-10, TB-7.7 & 71 & NE & {$[86]$} \\
\hline & & & & $P C-E C$ & 91 & $\mathrm{NE}$ & \\
\hline & & & & $\mathrm{PC}-\mathrm{HP}$ & 95 & $\mathrm{NE}$ & \\
\hline & African buffalo & $\mathrm{N}$ & $51+70$ & ESAT-6, CFP-10, TB-7.7 & 80.4 & 100 & {$[88]$} \\
\hline \multirow[t]{5}{*}{ IP-10 assay } & African buffalo & $\mathrm{N}$ & $44+40$ & PC-HP & 93.2 & 90 & {$[86]$} \\
\hline & & & & $P C-E C$ & 81.8 & 92.5 & \\
\hline & & & & ESAT-6, CFP-10, TB-7.7 & 86.4 & 92.5 & \\
\hline & African buffalo & $\mathrm{N}$ & $32+39$ & ESAT-6, CFP-10, TB-7.7 & 94 & 92 & {$[87]$} \\
\hline & African buffalo & $\mathrm{N}$ & $51+70$ & ESAT-6, CFP-10, TB-7.7 & 88.2 & 100 & {$[88]$} \\
\hline MAPIA & Wild bison & N & $12+70$ & M. bovis antigens ${ }^{\mathrm{a}}$ & 92 & 97 & {$[111]$} \\
\hline \multirow[t]{2}{*}{ FPA } & Wild bison & N & ND & MPB70 & 4.4 & 83.2 & {$[105]$} \\
\hline & Wild bison & $N$ & $9+57$ & MPB70 & 67 & 34 & {$[111]$} \\
\hline Idexx ELISA & African buffalo & $N$ & 8 & bPPD & 37.5 & $\mathrm{NE}$ & {$[7]$} \\
\hline BovidTB STAT-PAK & African buffalo & N & $100+100$ & ESAT-6, CFP-10, MPB83 & 33 & 90 & {$[114]$} \\
\hline VetTB STAT-PAK & Wild bison & $\mathrm{N}$ & ND & ESAT-6, CFP-10, MPB83 & 12.7 & 98.4 & {$[105]$} \\
\hline $\mathrm{RT}^{1}$ & African buffalo & $\mathrm{N}$ & $100+100$ & NM & 23 & 94 & {$[114]$} \\
\hline $\mathrm{RT}^{2}$ & Wild bison & $\mathrm{N}$ & $12+70$ & ESAT-6, CFP-10, MPB83 & 67 & 99 & {$[111]$} \\
\hline
\end{tabular}

N/E: natural or experimental infection; $n_{\mathrm{Se}}$ : number of TB positive animals used for evaluation of Se; $\mathrm{n}_{\mathrm{Sp}}$ : number of TB negative animals used for evaluation of Sp; Se: sensitivity; Sp: specificity; CFT: caudal fold test; SCITT: single comparative intradermal tuberculin skin test; IGRA: interferon gamma release assay; IP-10: interferon gamma-inducible protein 10; PC-EC: contains ESAT-6- and CFP-10-derived peptides; PC-HP contains ESAT-6- and CFP-10-derived peptides and 4 mycobacterial antigens (including Rv3615); MAPIA: multiantigenimmunoprint assay; FPA: fluorescent polarization assay; ELISA: enzyme linked immunosorbent assay; Idexx ELISA: commercial ELISA (Idexx Laboratories, Inc., Westbrook, ME, USA); Bovid TB STAT-PAK and DPP Vet TB: lateral flow tests (Chembio Diagnostic Systems, Inc., USA); RT ${ }^{1}$ : rapid test (Anigen, Animal Genetics, Inc., South Korea-name of the test not mentioned); RT²: lateral flow rapid test (Chembio Diagnostic Systems, Inc., USA); NE: not estimated; NM: not mentioned; ND: not defined, Se and Sp estimated based on Bayesian analysis (absence of gold standard).

a ESAT-6, CFP-10, MPB59, MPB64, MPB70, MPB83, the16-kDa protein, the 38-kDa protein, two fusion proteins comprising CFP-10/ESAT-6 and the 16 kDa protein/ MPB83, and two native antigens, bovine PPD and M. bovis culture filtrate.

cells are detected with biotinylated monoclonal antibody and the ELISPOT results are expressed as number of spot forming units/million cells. The main limitations of this technique are economical and technical as the samples must be collected in live anesthetized badgers and must be processed immediately.

\section{IFN $\gamma$-inducible protein 10 (IP-10) assay}

IFN $\gamma$-inducible protein 10 (IP-10) is a chemokine induced by IFN $\gamma$ which plays a role in type IV hypersensitivity reactions. The assay protocol is the same as that of IGRA, involving the stimulation of whole blood with mycobacterial antigens and the quantification of IP-10 by a sandwich ELISA. IP-10 is found to be a biomarker for the diagnosis of TB in African buffalo [85-87], warthog [89] and wild meerkat [90]. Most of the studies were conducted in African buffalo using bovine IP-10 antibodies (capture and detection antibody) either by a conventional ELISA (using bPPD or PC-EC or PC-HP) or by mQFT system (ESAT-6, CFP-10, TB7). The test has an excellent agreement with IGRA and it is reported to provide more diagnostic accuracy in comparison to conventional IGRA in African buffalo [86, 88]. In addition, it has high thermal stability that would facilitate the heat-inactivation of plasma pathogens and for the safe transport of diagnostic samples [87]. Preliminary studies in warthog and wild meerkat show IP-10 assay as a promising method, but the Se was low in warthog $[89,90]$.

\section{Lymphocyte stimulation test (LST)}

This test measures the reactivity of blood lymphocytes to mycobacterial antigens. The PBMCs are stimulated by mycobacterial antigens along with controls (Concanavalin A as positive control and fetal bovine serum or unstimulated medium as negative control) followed 
Table 5 Details of immunological diagnosis in elephants.

\begin{tabular}{|c|c|c|c|c|c|c|c|}
\hline Assay test & Species & N/E & $n_{s e}+n_{s p}$ & Antigens & Se (\%) & Sp (\%) & References \\
\hline Skin test ${ }^{b}$ & Asian elephant & $\mathrm{N}$ & $6+31$ & bPPD alone or with aPPD & 16.7 & 74.2 & [65] \\
\hline \multirow[t]{2}{*}{ Elephant TB Stat-Pak } & African/Asian elephant & $\mathrm{N}$ & $26+147$ & ESAT-6, CFP-10, MPB83 & 100 & 95.2 & [10] \\
\hline & African/Asian elephant & $\mathrm{N}$ & 14 & ESAT-6, CFP-10, MPB83 & 100 & $\mathrm{NE}$ & [122] \\
\hline MAPIA & African/Asian elephant & $\mathrm{N}$ & $26+147$ & M. bovis antigens ${ }^{c}$ & 100 & 100 & [13] \\
\hline \multirow[t]{2}{*}{ DPP VetTB assay } & African/Asian elephant & $\mathrm{N}$ & $26+147$ & ESAT-6, CFP-10, MPB83 & 100 & 100 & {$[10]$} \\
\hline & African/Asian elephant & $\mathrm{N}$ & 14 & ESAT-6, CFP-10, MPB83 & 100 & NE & [122] \\
\hline \multirow[t]{8}{*}{ ELISA } & African/Asian elephant & $\mathrm{N}$ & $7+40$ & CF & 100 & 95 & [98] \\
\hline & & & & bPPD & 85.7 & 97.5 & \\
\hline & & & & MPB70 & 28.6 & 97.5 & \\
\hline & & & & ERD & 71.4 & 85 & \\
\hline & & & & RA & 57.1 & 90 & \\
\hline & Asian elephant & $\mathrm{N}$ & $8+41$ & RA, CF, bPPD MPB70, ERD, aPPD & $100^{\mathrm{a}}$ & $87.8^{\mathrm{a}}$ & [65] \\
\hline & & & $3+25$ & $C F, L A M, a P P D$ & $33.3^{\mathrm{a}}$ & $100^{\mathrm{a}}$ & \\
\hline & & & $8+55$ & $\begin{array}{l}N \text {-lauryl-sarcosyl extract of M. bovis, } \\
\text { bPPD, aPPD, and M. intracellulare PPD }\end{array}$ & $87.5^{\mathrm{a}}$ & $83.6^{\mathrm{a}}$ & \\
\hline BTB & Asian elephant & $\mathrm{N}$ & $6+31$ & bPPD, aPPD & 83.3 & 51.6 & [65] \\
\hline
\end{tabular}

N/E: natural or experimental infection; $n_{\mathrm{Se}}$ : number of TB positive animals used for evaluation of $\mathrm{Se} ; \mathrm{n}_{\mathrm{Sp}}$ : number of TB negative animals used for evaluation of $\mathrm{Sp}$; Se: sensitivity; Sp: specificity; ELISA: enzyme linked immunosorbent assay; Elephant TB Stat-Pak and DPP VetTB assay: lateral flow rapid tests (Chembio Diagnostic Systems, Inc., USA); MAPIA: multiantigenimmunoprint assay; CF: M. bovis AN5 culture filtrate; ERD: lipoarabinomannan antigen Erdman strain of M. tuberculosis; RA lipoarabinomannan antigen H37 Ra strain of M. tuberculosis; LAM: lipoarabinomannans; BTB: blood tuberculosis test.

a Se and Sp are determined excluding the weak responses.

b Single intradermal tuberculin test, caudal fold test as well as single comparative intradermal tuberculin skin test depending on the herd.

c ESAT-6, CFP-10, MPB59, MPB64, MPB70, MPB83, the16-kDa protein, the 38-kDa protein, CFP-10/ESAT-6 and the 16 kDa protein/MPB83, bovine PPD and M. bovis culture filtrate.

Table 6 Details of immunological diagnosis in African lion.

\begin{tabular}{|c|c|c|c|c|c|c|}
\hline Assay test & N/E & $n_{s e}+n_{s p}$ & Antigens & Se (\%) & $\mathrm{Sp}(\%)$ & References \\
\hline SIT & N & $\begin{array}{l}52+32 \\
44\end{array}$ & bPPD & $\begin{array}{l}86.5 \\
N E\end{array}$ & $\begin{array}{l}81.2 \\
100^{a}\end{array}$ & [62] \\
\hline \multirow[t]{2}{*}{ SCITT } & $\mathrm{N}$ & $\begin{array}{l}52+32 \\
44\end{array}$ & bPPD, aPPD & $\begin{array}{l}80.8 \\
\text { NE }\end{array}$ & $\begin{array}{l}81.2 \\
100^{a}\end{array}$ & [62] \\
\hline & $\mathrm{N}$ & 8 & bPPD, aPPD & 63 & NE & [115] \\
\hline $\begin{array}{l}\text { Elephant } \\
\text { TB } \\
\text { Stat-Pak }\end{array}$ & $\mathrm{N}$ & 11 & ESAT-6, CFP-10, MPB83 & 64 & NE & [115] \\
\hline DPP Vet TB & $\mathrm{N}$ & 10 & ESAT-6, CFP-10, MPB83 & 70 & $\mathrm{NE}$ & [115] \\
\hline
\end{tabular}

N/E: natural or experimental infection; $\mathrm{n}_{\mathrm{Se}}$ : number of TB positive animals used for evaluation of Se; $\mathrm{n}_{\mathrm{Sp}}$ : number of TB negative animals used for evaluation of Sp; Se: sensitivity; Sp: specificity; SIT: single intradermal cervical test; SCITT: single comparative intradermal tuberculin skin test: NE: not estimated; Elephant TB Stat-Pak and DPP Vet TB are lateral flow rapid tests (Chembio Diagnostic Systems, Inc., USA).

a $\mathrm{Sp}$ of the test when 44 samples from uninfected population are considered and only 11 of which are tested negative by culture.

Table 7 Details of immunological diagnosis in possum.

\begin{tabular}{lllllll}
\hline Assay test & $\mathbf{N} / \mathbf{E}$ & $\mathbf{n}_{\mathbf{s e}}+\mathbf{n}_{\mathbf{s p}}$ & Antigens & Se (\%) & Sp (\%) & References \\
\hline ELISA & $\mathrm{N}$ & $29+100$ & M. bovis culture filtrate MBP70 & $45^{\mathrm{a}}$ & $96^{\mathrm{a}}$ & $98^{\mathrm{a}}$ \\
& & & $21^{\mathrm{a}}$ & $28^{\mathrm{b}}$ & $99^{\mathrm{b}}$ & {$[100]$} \\
RT & $\mathrm{N}$ & $38+91$ & ESAT-6, CFP-10, MPB83 & 44.7 & 85.7 & {$[109]$} \\
\hline
\end{tabular}

N/E: natural or experimental infection; $\mathrm{n}_{\mathrm{Se}}$ : number of TB positive animals used for evaluation of Se; $\mathrm{n}_{\mathrm{Sp}}$ : number of TB negative animals used for evaluation of Sp; Se: sensitivity; Sp: specificity; ELISA: enzyme linked immunosorbent assay; RT: lateral flow rapid test (name not mentioned- Chembio Diagnostic Systems, Inc., USA).

a Se or Sp of ELISA where blocking antibody against MBP70 is not used.

b Se or Sp of blocking ELISA where a monoclonal antibody against MPB70 is used. 
Table 8 Details of immunological diagnosis in wild meerkat.

\begin{tabular}{lllllr}
\hline Assay test & N/E & $\mathbf{n}_{\text {se }}+\mathbf{n}_{\text {sp }}$ & Antigens & Se (\%) & References \\
\hline MAPIA & $N$ & ND & M. bovis antigens ${ }^{*}$ & 90 & 48 \\
BovidTB STAT-PAK & $N$ & ND & ESAT-6, CFP-10, MPB83 & 43 & [9]
\end{tabular}

MAPIA: multiantigenimmunoprint assay; BovidTB STAT-PAK: lateral flow test (Chembio Diagnostic Systems, Inc., USA); N/E: natural or experimental infection; $\mathrm{n}_{\mathrm{Se}}$ : number of TB positive animals used for evaluation of Se; $\mathrm{n}_{\mathrm{Sp}}$ : number of TB negative animals used for evaluation of Sp; Se: sensitivity; Sp: specificity; ND: not defined, Se and $\mathrm{Sp}$ were estimated based on Bayesian analysis (absence of gold standard).

a ESAT-6, CFP-10, MPB59, MPB64, MPB70, MPB83, the16-kDa protein, the 38-kDa protein, CFP-10/ESAT-6 and the $16 \mathrm{kDa}$ protein/MPB83, bovine PPD and M. bovis culture filtrate.

by the addition of $\left[{ }^{3} \mathrm{H}\right]$-thymidine and incubation for 4 days. Lymphocyte stimulation is assessed by the uptake of $\left[{ }^{3} \mathrm{H}\right]$-thymidine and data are expressed in counts per minute (cpm). Usually, the difference in the OD of antigens with negative control and aPPD is taken into consideration for interpreting the results. The test has been performed in deer [68, 91], badger [92], possum [16] and elephant [65]. The use of MPB70 in red deer resulted in improved Sp, but low Se compared to LST using bPPD [68]. In badger, Se of LST was high, but Sp was low in comparison to ELISA [92]. Lymphocyte stimulation responses were detected in $93 \%$ of experimentally infected possums [16]. This method is complicated to perform in field conditions for screening large number of samples because of the time requirement and logistics. Hence, nowadays, it is not usually used for diagnostic purpose.

Quantitative reverse-transcription PCR assay ( $q R T-P C R$ ) The production of cytokines can be detected by measuring the relative level of gene expression using qRT-PCR. The assay includes reverse transcription of mRNA of cytokines of interest (IL-2, IL-4, IL-10, IL-12p40, IFN- $\gamma$, TNF- $\alpha$ ) into cDNA, followed by real-time PCR using species specific or cross reactive primers. The relative gene expression of IFN $\gamma$ in response to bPPD and specific antigens was found to be high in infected red deer, elk [84] or badger [93]. qRT-PCR can be used as an alternative to the current serological methods of diagnosis like the brock test in badger [93]. In red deer and elk, qRT-PCR is found to be superior to LST and Cervigam assay [84]. The assay can be exploited as a major diagnostic platform for use in wildlife, since primers are easier to develop as well as it is a simple, rapid, and sensitive measure of antigen-specific CMI and it does not need double handling of animals as in the case of skin test. The demerits include the need of quick processing of samples and requirement of costly reagents [84, 93].

Antibody based tests Antibody assays are convenient to perform as samples can be stored for prolonged time before processing. They ensure large scale screening of samples obtained ante-mortem and post-mortem and are able to diagnose the progressive disease [94]. In this regard, the Se increases in advanced stages of the disease.

\section{Enzyme-linked immunosorbent assay (ELISA)}

ELISA is the serodiagnostic technique most extensively used in wildlife. It detects circulating antibodies against MTC, and it is well suitable for large scale screening of the disease, both ante-mortem and post-mortem. There are reports of many in house ELISAs (indirect ELISA of bPPD/P22/LAM/M. bovis culture filtrate (MBCF)/ MPB83 and MPB70 in red deer, wild boar, warthog, African buffalo, elephant, possum and badger) or commercial ELISA kits (ELISA TB-VK, ELISA-INgezim TB porcine and INgezim Tuberculosis DR in wild boar and warthog and Idexx ELISA in wild bovids) using species specific anti-IgG antibodies or cross reactive antibodies like protein $\mathrm{G}$ (as conjugate) available for use in wildlife with varying levels of diagnostic accuracy [24, 25, 95-98] (see Tables 1, 2, 3, 4, 5, 6, 7 and 8). Among them, ELISA is the prime choice for TB diagnosis in swine [25], being bPPD the most common antigen used [24, 25, 68, 97, 98], but with low Sp due to cross reactivity with other nontuberculous mycobacteria. Serum diluted in skimmed milk supplemented with aPPD (competitive ELISA) was found to have more Sp in badger compared to the simple indirect ELISA [99]. Many other specific or purified antigens (LAM, MPB83, MPB70, ESAT-6 and CFP-10, and P22) have been tested to improve the Sp in wild boar [25], red deer [24, 68], wild bovids [7], badger [99] and possum [100]. However, consequently, in some studies the Se is compromised [80]. Brock test is an indirect ELISA based on MPB83 used for TB diagnosis in badger, which provided high Sp, but low Se [92, 96, 101]. Dachs TB-ELISA, also based on MPB83, has high Se, similar Sp and high predictive values compared to Brock test [95]. Nevertheless, in some other reports, similar or even high Se was achieved with specific or purified antigens in red deer and wild boar [24, 25]. The use of antigenic cocktails (MPB70, MPB83), ethanol extract of $M$. bovis antigen (EVELISA) and prior pre-absorption of M. avium 
subspecies paratuberculosis antibodies or antibodies to other environmental mycobacteria as $M$. phlei have also proven promising results in red deer [102]. In wild bovids, Se was low in Idexx ELISA which might be due to the limited sample size used. Moreover, TB is a slow and progressive chronic disease so that antibodies might be detected in the terminal stages only (i.e. buffaloes) [7]. In elephants, substantial humoral immune response was detected in positive animals which could help in differentiating positive and negative animals $[65,98]$. In possum, low Se was observed in antibody mediated diagnosis, possibly due to the fact that very few possums with sub-clinical M. bovis infection produce positive antibody responses [100].

\section{Fluorescence polarization assay (FPA)}

This test comprises the use of the target antigen MPB70 with a fluorescent molecule bound to it, in order to detect antibodies in serum. This assay was first described by Surujballi et al. (2002) in cattle [103], and later it has been validated in red deer [91], elk [104] and wild bison [105], but with comparatively low diagnostic value in wild bison and cervids when the test is used alone [104, 105]. The Se is very low in early stages of infection [75].

\section{Multiantigen print immunoassay (MAPIA)}

The MAPIA uses a panel of 12 mycobacterial antigens including eight purified recombinant proteins (ESAT-6, CFP-10, MPB64, MPB59, MPB70, MPB83, Acr1, and the $38 \mathrm{kDa}$ protein), two protein fusions (CFP-10/ESAT-6 and Acr1/MPB83), and two native antigens, such as bPPD and MBCF [106]. The assay enables the qualitative identification of species-specific immunodominant proteins as well as the reactivity patterns over the course of the disease which, in turn, helps in the selection of antigens for other diagnostic tests [106]. The MPB83 alone or in combination with the protein Acr1 was found to be the most serodominant antigen followed by ESAT-6/ CFP-10 in elk and fallow deer [107, 108], red deer-elk hybrids [75], white-tailed deer [108, 109] and possum [109]. MPB83 is also the most serodominant antigen in wild boar and warthogs [109, 110]. In badger, MPB83 and $\mathrm{MBCF}$ are the most serodominant antigens [101, 109] followed by MPB70 [101] or CFP-10/ESAT-6 [109]. ESAT-6 alone is the most serodominant protein in wild bison [111], while ESAT-6 and CFP-10 are in elephant $[10,108]$ and black rhinoceros [112]. The MAPIA gave almost equal or even high Se and Sp in comparison to other rapid tests, ELISA or immunoblot, but there is a practical difficulty to implement this assay for screening large number of samples $[55,101]$.

\section{Immunoblotting (IB)}

Electrophoresis and immunoblot are qualitative assays, usually performed using whole cell sonicate antigen in reindeer [55], white-tailed deer [113] and elephant [48], but it is not routinely used for diagnosis in any species. Instead, the test is used to confirm that the real antibodies are detected in other serodiagnostic tests like ELISA/ MAPIA/immunochromatographic test.

\section{Lateral flow tests}

These tests are based on the immunochromatography. Lateral flow test kits have great practical applicability in wildlife because of its easiness to perform and immediate test results, although their Se is limited. Most of the lateral flow tests are qualitative except DPP test. The main lateral flow diagnostic tests are listed below:

- TB STAT-PAK (Chembio Diagnostic Systems, Inc., Medford, NY). This test employs a unique cocktail of MPB83, ESAT-6 and CFP10 antigens, with a singlestrip bead-based signal detection system [75]. The use of this test has been reported in multiple species, i.e. Cervid TB STAT PAK in deer [75], Brock TB STAT-PAK in badger [96], Bovid TB STAT PAK in African buffalo [114] and wild meerkat [9], TB STAT PAK in pygmy hippopotamus [68] and elephant TB STAT PAK in elephant [10], lion [115], black rhinoceros [112] and banded mangoose [116]. Its advantages include easiness to perform in field with a small volume of blood, serum or plasma, and possibility to detect immunoglobulin (Ig) A (IgA), IgM and IgG antibodies to MTC [117]. The test has also legitimate diagnostic accuracy in most of the species tested. However, the performance of test was poor in African buffalo as in the case of ELISA [114] and showed poor Se in wild meerkat when the test was used alone [9]. In comparison to DPP test, false positive results can occur due to the presence of non-tuberculous mycobacteria or inflammatory conditions in elephant [118], as well as false negative results may happen due to the limited Se [52].

- DPP tests (Chembio Diagnostic Systems, Inc., Medford, NY). This assay has two test antigen bands on the membrane strip, T1 (MPB83 protein) and T2 (CFP-10/ESAT-6 fusion), for differential IgG antibody detection by colloidal gold particles coupled with hybrid protein $A / G$, in contrast to the 
single-strip format used in the TB STAT-PAK test [10]. One of the DPP tests is DPP Vet TB in which the presence and intensity of either of the two separate test lines (MPB83 antigen and CFP10/ESAT-6 fusion protein) are evaluated visually and by a DPP optical reader [10]. The DPP Vet TB has been employed in multiple species of deer [119-121], wild suids [110], elephant [10, 122] and badger [123]. The DPP Vet TB has higher Sp than STAT PAK in elephant [10] and deer [119]. The DPP VetTB assay is approved by the United States Department of Agriculture (USDA) Bovine TB Eradication Program for testing several species of captive cervids $[108,121]$ and it is considered appropriate for use in a badger bovine TB control campaign in Northern Ireland [123]. Another test based on DPP technology is DPP WTB which makes use of two antigens in separate test lines (MPB83 and MPB70). This assay is mainly focused for diagnosis in suidae, since MPB70 antigen is more serodominant than CFP-10/ESAT-6 in suids and it is found to be more sensitive than DPP Vet TB in wild boar [124]. The DPP bovid TB is another kind of test based on CFP-10/ESAT-6 and MPB70/ MPB83 chimeric antigens, but it had low Se in wild bovids which is consistent with reports of other serological assays in wild bovids.

- INgezim TB-CROM Ab (INGENASA S.A., Madrid, Spain). This is a recently developed test in which INgezim TB-CROM Ab uses MPB83 antigen. In wild boar, test acquired high diagnostic value, as well as concordance with ELISA (in house ELISA, commercial ELISAs- INgezim TB porcine, INgezim Tuberculosis DR) so that INgezim TB-CROM Ab can be used as a first approach for the surveillance of $\mathrm{TB}$ in this species [125].

Other tests Blood tuberculosis test (BTB) is a composite test, comprising of ELISA reactivity towards mycobacterial antigens as well as lymphocyte stimulation [65]. The test has been employed in red deer [126] and elephant [65]. However, this test is no longer in use because of its complexity to perform, especially in elephants, in which multiple tests can delay the treatment protocol [65]. Another biomarker, monocyte chemoattractant protein (MCP)-1, could also be a sensitive marker for TB diagnosis in African buffalo [85]. Gene expression assay of the chemokine (C-XC motif) ligand 9 (CXCL9) is a useful tool for the determination of $M$. bovis status in free-ranging lions [127]. Analysis of volatile organic compounds (VOCs) obtained from breath and feces by electronic nose or gas chromatography-mass spectrometry (GC-MS) is a promising tool for noninvasive detection of TB in badger [128], white-tailed deer [129] and wild boar [130].

\section{Confounding factors}

There are many factors related to host, environment, sampling and diagnosis technique which can affect the performance of the TB diagnostic test:

\section{Host}

Reports are available about the effect of age and sex on diagnostic accuracy in some species. There are no evident age-related differences in red deer and fallow deer in the responsiveness to skin test [21]. Males had greater response than females in skin test in cervids and this gender based difference was more evident with increasing age [21]. This difference could be due to the differences in reproductive effort and energy expenditure [131]. Moreover, males tend to have a thicker skin than females, so skin fold increase is related to the thickness of the skin in red deer [132] and fallow deer [21]. In wild boar, an increase in skin responsiveness with age was noticed, but there was no sex by age interaction [22]. Serodiagnostic techniques in white-tailed deer showed no age or sex related differences (Immunoblot, MAPIA, ELISA, CervidTB STAT-PAK) [113]. In wild boar, infected piglets had lower Se in ELISA and DPP tests, as compared to yearling, juvenile or adult wild boar [124, 125]. Gender-based variation was not significant in serodiagnostic tests like ELISA and DPP test in wild boar [133]. In badger, Se of IGRA was low in cubs compared to adults, but Sp of IGRA, Brock test and Brock TB STAT-PAK was high in cubs compared to adults [96]. The immunological status of the host needs to be considered, as anergic animals do not respond to diagnostic tests [23].

\section{Environment, habitat and management}

Skin responsiveness to mitogen in winter was found to be significantly higher compared to the response in summer and the difference was more prominent in adult red deer [132]. This may be due to seasonal presence of non-tuberculous mycobacteria, which is a main confounding factor in TB diagnosis in all species, especially in ruminants like deer [77] and wild bovids [134]. Sp of the serological assays varied between badger populations/countries of origin (higher Sp in badgers of Spain i.e. $96.88-100 \%$ compared to those of Republic of Ireland i.e. $85.7 \%$ ) possibly as a result of variable exposure to different environmental mycobacteria species [99]. Also, the type of management practices which determine the exposure to non-tuberculous mycobacteria could be another 
factor resulting in the variation of Sp (high Sp in intensive management compared with extensive management in suids) [25].

\section{Prior sensitization, history and other infections}

The preliminary skin testing for $\mathrm{TB}$ can also lead to the diagnosis of false positive animals with other diagnostic techniques [126]. Se of ELISA was considerably increased in red deer 10 days after skin test compared to the Se before skin test ( $45.7 \%$ to $85.3 \%$ in ELISA) [126]. In experimental infection in white-tailed deer, reindeer, red deer and black rhinoceros, an elevated antibody response (in ELISA, immunoblot analysis, MAPIA and rapid tests) could be detected shortly after skin testing $[55,75,112$, 119]. However, assays of repeated comparative skin testing in red deer at 6 months interval confirmed that it did not affect serological results [78] and lower IFN $\gamma$ response was detected in TB positive African buffalo after skin test [66]. On the other hand, past exposure to MTC/ $B C G$ vaccination can result in false positive reactions in red deer [135], whereas cross reactions with other nontuberculous mycobacteria could be a factor affecting the Sp of both cell mediated and humoral tests in several species $[24,77]$.

\section{Sample and sampling related factors}

The in vitro production of IFNY in IGRA and IGRA ELISPOT is influenced by the blood storage temperature and duration of storage until processing (recommended storage at room temperature and maximum time for processing is $8 \mathrm{~h}$ ), as well as by the type of anticoagulant used (optimal response with heparin) [52, 72, 80]. CMI based tests require fast processing of the sample, while appropriately stored samples can be used in antibody based diagnosis. CMI response measured in vitro is significantly reduced in blood taken immediately after death of the animal [80]. However, overnight stored blood samples had improved Sp in LST in badger without any change in Se (Sp 100\% in samples stored overnight and $84.6 \%$ in fresh samples) [92]. Incubation of plasma at $65^{\circ} \mathrm{C}$ for 20 min or plasma stored on Protein Saver Cards for 2 and 8 weeks did not cause any considerable loss of IP-10 concentration in IP-10 assay which allows the short term storage and transport of samples [87]. In a similar way there was no change in the IFN $\gamma$ production in samples in which maintenance media (RPMI-1640 medium containing fetal calf serum L-glutamine and penicillin-streptomycin) was added and stored at $4{ }^{\circ} \mathrm{C}$ [72]. For humoral-based tests, the antibody responses varied between the type of the sample, i.e. serum, plasma, fresh whole blood, diaphragm fluid and aqueous humor [109, 123]. The source of the sample can also cause variation in diagnostic results, observing that serological test results for hunter or veterinarian harvested blood samples had higher level of agreement with culture results than samples from carcasses with TBL [113]. Repeated freezethawing cycles, delay in shipping and occurrence of haemolysis could possibly affect the results of these serological tests [48]. Thus, high levels of haemolysis decreased the Se of antibody tests, being more evident for the bPPD ELISA, but not affecting the results of rapid tests [113], which are designed not only for serum, but also for whole blood.

\section{Diagnostic technique related factors}

In all methodologies of TB diagnosis, the Se and Sp greatly depends on the type of antigen used, improving the diagnostic accuracy through the use of specific or purified antigens (see Tables 1, 2, 3, 4, 5, 6, 7 and 8). In skin test, there can be technical variation in results depending on the site of inoculation, dose and potency of tuberculin administered and reproducibility between operators [76]. There can be variation in Se depending on the type of assay used to measure the IFNY released (ELISA/ELISPOT) [136] or the use of monoclonal pairs (mEIA) or polyclonal antiserum (pEIA) against IFN $\gamma$ (80.9\% Se for mEIA, 74.5\% for pEIA) [80]. In the humoral diagnostic tests, the conjugate used could also influence Se, obtaining better performance in ELISA with species-specific conjugate (anti-IgG pig- $46.2 \% \mathrm{Se}$ ) than with protein G conjugate (23.1\% Se) in wild boar [124]. In quantitative tests, their interpretation depends on the selected cut off value which, in turn, depends on the preferred outcome of the test (better Se or Sp or better compromise between Se and $\mathrm{Sp}$ ) because Se increases at the expense of Sp and vice versa [81,137].

Overall, reference tests used have a major influence on the validated diagnostic tests. The gold standard test, mycobacterial culture, has been widely used in validation; however, it can have variable Se and heavy reliance on the number and quality of tissues examined at necropsy [104, 133]. In some studies, skin test or presence of TBL or IGRA or STAT PAK or histopathology were used alone or in combination as reference standards for deer $[68,120,138]$, badger [139] and wood bison [111]. In case of wildlife, culture of samples has little practical applicability as far as the difficulty in collection and processing are concerned. Hence, now culture has been considered as an imperfect gold standard for validation of new diagnostic tests in wildlife, as it may underestimate the Se and Sp of newer diagnostic tests being validated $[9,104]$. In this regard, Bayesian analytical technique is proved to be a good alternative for estimating the diagnostic accuracy of tests $[104,105]$. 


\section{Improved diagnosis}

\section{Selection of the appropriate test}

The selection of an appropriate test is based on many factors like species being tested, stage of the disease, diagnostic accuracy of the test, economic feasibility, ease of performing the test, as well as purpose of diagnosis. Skin test is usually employed in deer and wild bovids $[63,68]$, but it has limited application in other species like suidae, elephant, badger, possum or lion $[22,62,65,74]$. The stage of the disease progression is a major factor involved in the selection of a test, being CMI-based tests more accurate in identifying the early stages of infection in contrast to antibody-based tests which are more useful in later stages than in early ones $[23,24]$. Moreover, in the antibody tests, the antigen MPB83 is detected early in the course of experimental MTC infections [55], unlike MPB70, which elicits a humoral response to $\mathrm{MTC}$ in the later stages of the disease [140]. On the other hand, CMI tests, especially skin test, IGRA and IGRA ELISPOT can only be applied for the diagnosis of live animals, while antibody-based can be applied to both live and dead animals [25]. Among the latter, the ELISA techniques are useful for evaluating a large number of samples [25] whereas rapid tests are easy to perform (also in field conditions) and give rapid results compared to any other test [119]. However, rapid tests are not economically viable for screening large number of field samples. In addition to all, it is important to consider the objective intended to choose a diagnostic test for the detection of $\mathrm{TB}$, so a test with high Se is required when the diagnosis is performed for detection of the maximum number of positive animals [104], while a high $\mathrm{Sp}$ is the major factor when the test is done for test and slaughter procedures in order to avoid false positive reactors and thereby a huge economic loss [77].

\section{Proper implementation and interpretation of the test}

Proper implementation of the test procedure is especially important to minimize the diagnostic errors and for better results. The Se and Sp are the major factors evaluated for assessing the test result, being a highly sensitive test usually related to a low $\mathrm{Sp}$ and vice versa [137]. Thus, a higher cut off value minimizes the chance of false positives, but lower cut off values enable the maximum identification of infected animals [99, 137]. In addition, predictive values, likelihood ratio and diagnostic odds ratio have importance in interpreting the results [70]. The prevalence of disease must be taken into account for interpreting the result, since a higher prevalence tends to lead to an increased positive predictive value (PPV) and a decreased negative predictive value (NPV), whilst a lower prevalence tends to lead to an increased NPV and a decreased PPV [141].

\section{Combination of different diagnostic tests or different antigens}

Combination of two diagnostic test results in parallel $[40,71,104,142]$ or in series $[21,142]$ can enhance

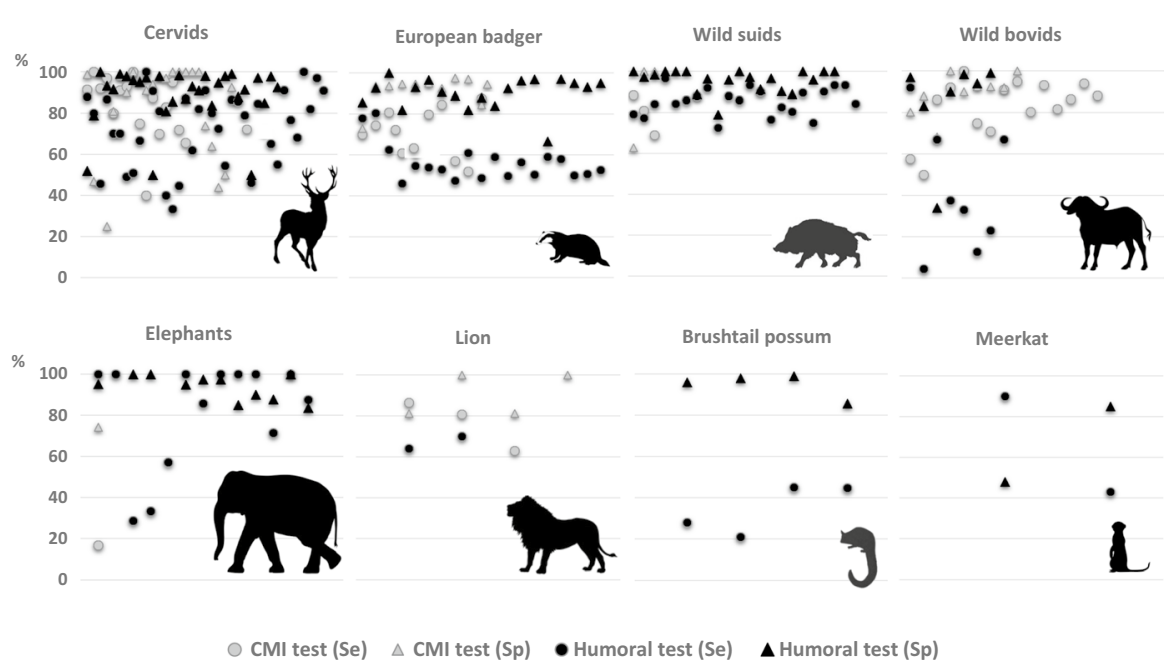

Figure 3 Sensitivity (Se) and specificity (Sp) of immunological tests used in wildlife tuberculosis diagnosis in cervids ( $\mathrm{n}=108$ evaluations), European badger $(n=59)$, wild suids $(n=48)$, wild bovids $(n=39)$, elephants $(n=28)$, lion $(n=9)$, brushtail possum $(n=8)$ and meerkat $(\mathbf{n}=\mathbf{4})$. Details can be consulted in Tables 1, 2, 3, 4, 5, 6, 7 and 8. CMl: diagnostic methods based on cell-mediated immunity. 
the diagnostic accuracy. Parallel testing is a method in which two screening tests are performed at the same time and the results are subsequently combined, resulting in higher Se but lower Sp. Serial testing means that both techniques can be performed sequentially; if the result of the first screening test is positive (serial testing), the second screening test will be performed to avoid false positive results [104]. Usually, CMI-based tests are interpreted in parallel to antibody-based tests resulting in very high Se which aids to detect a maximum number of animals in different stages of the disease, thereby facilitating test and removal strategies for the disease control in wildlife. Thus, the combination of Bovigam assay (bPPD antigen) and SCITT/IGRA/Idexx ELISA or Bovigam assay (bPPD, PC-EC, PC-HP) yielded $100 \%$ Se in parallel interpretation in African buffalo, while the second Bovigam assay alone offered a Se of 75\% [7]. Similarly, the parallel use of SCITT with Bovigam assay or IP-10 assay was also able to identify all the infected animals in African buffalo [71]. In the same way, parallel interpretation of SCITT and Cervid TB STAT-PAK allowed the detection of all $M$. bovis confirmed by culture in fallow deer [21], and the parallel use of FPA, LST and Cervid TB STAT-PAK identified all the infected elk [104]. Pathological lesions and culture results were interpreted in series to determine true infection status in fallow deer for minimizing the number of false positives [104]. Combination of Brock TB STAT PAK with IGRA and culture allowed a diagnosis to be made for individual animals with an estimated overall accuracy of $93 \%$ in badger $[142,143]$.

The use of multiple antigens in the same diagnostic test (IGRA, ELISA or rapid tests) and its further interpretation improved the diagnostic accuracy. In red deer, the evaluation in parallel of ESAT-6/CFP-10 with Rv3615c and Rv3020c antigens increased the Se of the technique when compared to the separate use of these antigens [23]. Moreover, multi-antigen ELISA also enhanced Se of TB diagnosis in badger [95] and elephant [65, 98].

\section{Conclusions}

Culture and identification of MTC remain as the gold standard method in wildlife TB diagnosis, even so limited Se and little practical application exists as far as the difficulty in collection and processing of samples are concerned. In this regard, pathological studies can help to increase the culture Se. In recent years there has been considerable progress in wildlife TB diagnosis, where cellular and humoral immunological diagnostic tests are gaining importance, mainly in cervids, badgers, wild bovids and wild suids (Figure 3). Regarding cellular based diagnostic techniques, SIT is the official ante-mortem test in many species, especially in cervids; however, the development of IGRA offers an improvement in the diagnostic accuracy not only in cervids, but also in African buffalo or badgers. Serological tests are especially useful in wildlife because they are economically attractive, technically easy, the large-scale surveillance is possible in a short period of time and tests can be applied either in live or dead animals. Lateral flow tests are very convenient for use in wildlife since they are effortless to perform and give rapid results; however, Se and Sp of these tests must be still improved. Variability in the Se and $\mathrm{Sp}$ of the same technique has been observed according to the target species (i.e. SIT in cervids and badgers) and, therefore, the testing strategy should be also adapted to the target species, as well as to the logistic and budget constraints. According to the information collected, serological tests for MTC-antibody detection are especially important in wildlife, since the possibility of being used together with the post-mortem examination supposes a sensitive and cost-effective means of disease surveillance that should be maintained or implemented; while tests based on CMI are still relevant in wild ruminants. In farmed or easily handled wild animals, it is important to highlight that combinations of cellular and humoral tests could enhance the diagnostic accuracy, since animals in different stages of the disease would be detected. Future studies are still needed in the area of wildlife TB diagnosis in order to reach an accurate, rapid and cost-effective diagnosis in target species. Moreover, testing must be consistent over space and time to allow proper disease monitoring.

\section{Supplementary Information}

The online version contains supplementary material available at https://doi. org/10.1186/s13567-020-00881-y.

Additional file 1. Search algorithms used for collection of articles. Different algorithms used in collection of data for systematic review are explained.

Additional file 2. An overview of tuberculosis (TB) diagnostic tests in wildlife.

\section{Abbreviations}

aPPD: Avian purified protein derivative; BAL: Bronchoalveolar lavage; BCG: Bacillus calmette guerin; bPPD: Bovine purified protein derivative; BTB: Blood tuberculosis test; CFP-10: Culture filtrate protein-10 kDa; CFU: Colony forming unit; CMI: Cell-mediated immunity; DIVA: Differentiation of infected and vaccinated animals; DPP: Dual path platform; ELISA: Enzyme-linked immunosorbent assay; ELISPOT: Enzyme-linked immunospot assay; ESAT-6: Early secretory antigenic target-6 kDa; FPA: Fluorescence polarization assay; IB: Immunoblotting; IFNy: Interferon gamma; Ig: Immunoglobulin; IGRA: IFNy response assay; IL: Interleukin; IP-10: IFNy-inducible protein 10; LAM: Lipoarabinomannan; LN: Lymph node; LST: Lymphocyte stimulation assay; MAC: Mycobacterium avium complex; MAPIA: Multiantigen print immunoassay; MBCF: Mycobacterium bovis culture filtrate; MPB 83: Mobility protein of M. bovis 83; MPB 70: Mobility protein of M. bovis 70; mQFT: Modified QuantiFERON ${ }^{\circledR}$ TB gold in-tube; MTC: Mycobacterium tuberculosis complex; NPV: Negative predictive value; OD: Optical density; OIE: Office international des epizooties; PBMC: Peripheral blood mononuclear cells; PBS: Phosphate buffered saline; PCR: Polymerase chain reaction; PPV: Positive predictive value; PRISMA: Preferred reporting 
items of systematic reviews and meta-analyses; qRT-PCR: Quantitative reversetranscription polymerase chain reaction assay; RPMI: Roswell Park Memorial Institute medium; SCITT: Comparative intradermal tuberculin skin test; Se: Sensitivity; SIT: Single intradermal tuberculin skin test; Sp: Specificity; TB: Tuberculosis; TBL: TB like lesions.

\section{Acknowledgements}

Authors thank the Spanish Ministry of Agriculture, Fisheries and Food (MAPA).

\section{Authors' contributions}

Conceptualization, MAR and CG; validation, MAR and AB; resources, AB and CG; data curation, JT and MAR; writing —original draft preparation, JT; writing — review and editing, $A B, C G$ and MAR; visualization, JT, $A B$ and MAR; supervision, MAR and CG; project administration, CG; funding acquisition, $A B$ and CG. All authors read and approved the final manuscript.

\section{Funding}

This work has been funded by project MYCOTRAINING SBPLY/19/180501/000174 (Junta de Castilla-La Mancha), Agencia Estatal de Investigación grant WildDriver CGL2017-89866 (MINECO, Spain and EU FEDER), project RTI2018-096010-B-C21 (MCIU and AEl; FEDER co-funded), and PCTI 2018-2020 (GRUPIN: IDI2018-000237) (Gobierno del Principado de Asturias and FEDER). J. Thomas was supported by a grant from the Indian Council of Agricultural Research-International Fellowship 2014-2015 (ICAR-IF 2014-2015).

\section{Availability of data and materials}

The data and materials will be available upon request

\section{Ethics approval and consent to participate}

Not applicable.

\section{Consent for publication}

Not applicable.

\section{Competing interests}

The authors declare no competing interests.

\section{Author details}

${ }^{1}$ Sanidad Y Biotecnología (SaBio), Instituto de Investigación en Recursos Cinegéticos IREC (UCLM-CSIC), 13003 Ciudad Real, Spain. ${ }^{2}$ Indian Council of Agricultural Research (ICAR), New Delhi 110001 , India. ${ }^{3}$ Departamento de Sanidad Animal, Facultad de Veterinaria, Universidad de León, 24071 León, Spain. ${ }^{4}$ Departamento de Sanidad Animal, Instituto de Ganadería de Montaña (CSIC-Universidad de León), Finca Marzanas, Grulleros, 24346 León, Spain. ${ }^{5}$ Departamento de Anatomía Y Anatomía Patológica Comparadas Y Toxicología. Facultad de Veterinaria, Universidad de Córdoba (UCO), 14014 Córdoba, Spain. ${ }^{6}$ Unidad de Enfermedades Infecciosas, Grupo de Virología Clínica Y Zoonosis, Instituto Maimónides de Investigación Biomédica de Córdoba (IMIBIC), Hospital Reina Sofía, Universidad de Córdoba (UCO), 14004 Córdoba, Spain.

Received: 5 August 2020 Accepted: 14 December 2020 Published online: 24 February 2021

\section{References}

1. Caminiti E (2019) The socio-economic costs of bovine tuberculosis. https://oiebulletin.com/?panorama=3-05-tb-costs-en. Accessed 15 Mar 2020.

2. Gortázar C, Che Amat A, O'brien DJ (2015) Open questions and recent advances in the control of a multi-host infectious disease: animal tuberculosis. Mammal Rev 45:160-175

3. Gortázar C, Fernández-Calle LM, Collazos-Martínez JA, MínguezGonzález O, Acevedo P (2017) Animal tuberculosis maintenance at low abundance of suitable wildlife reservoir hosts: a case study in northern Spain. Prev Vet Med 146:150-157

4. Nigsch A, Glawischnig W, Bagó Z, Greber N (2019) Mycobacterium caprae infection of red deer in Western Austria-optimized use of pathology data to infer infection dynamics. Front Vet Sci 5:350
5. Santos N, Richomme C, Nunes T, Vicente J, Alves PC, de la Fuente J, Correia-Neves M, Boschiroli ML, Delahay R, Gortázar C (2020) Quantification of the animal tuberculosis multi-host community offers insights for control. Pathogens 9:421

6. de Garine-Wichatitsky M, Caron A, Kock R, Tschopp R, Munyeme M, Hofmeyr M, Michel A (2013) A review of bovine tuberculosis at the wildlife-livestock-human interface in sub-Saharan Africa. Epidemiol Infect 141:1342-1356

7. Van der Heijden EMDL, Jenkins AO, Cooper DV, Rutten VP, Michel AL (2016) Field application of immunoassays for the detection of Mycobacterium bovis infection in the African buffalo (Syncerus caffer). Vet Immunol Immunopathol 169:68-73

8. Roos EO, Olea-Popelka F, Buss P, Hausler GA, Warren R, Van Helden PD, Parsons SD, de Klerk-Lorist LM, Miller MA (2018) Measuring antigen-specific responses in Mycobacterium bovis-infected warthogs (Phacochoerus africanus) using the intradermal tuberculin test. BMC Vet Res 14:360

9. Drewe JA, Dean GS, Michel AL, Pearce GP (2009) Accuracy of three diagnostic tests for determining Mycobacterium bovis infection status in live-sampled wild meerkats (Suricata suricatta). J Vet Diagn Invest 21:31-39

10. Greenwald R, Lyashchenko O, Esfandiari J, Miller M, Mikota S, Olsen JH, Ball R, Dumonceaux G, Schmitt D, Moller T, Payeur JB (2009) Highly accurate antibody assays for early and rapid detection of tuberculosis in African and Asian elephants. Clin Vaccine Immunol 16:605-612

11. Chileshe J, Roos EO, Goosen WJ, Buss P, Hausler G, Rossouw L, Manemela T, van Helden P, Warren R, Parsons SD, Miller M (2019) An interferon-gamma release assay for the diagnosis of the Mycobacterium bovis infection in white rhinoceros (Ceratotherium simum). Vet Immunol Immunopathol 217:109931

12. Roux L, McCall AJ, Michel AL (2019) Detection of native interferon- $y$ in nyala (Tragelaphus angasii): towards diagnosing tuberculosis. Onderstepoort J Vet Res 86:1-3

13. Viljoen IM, Sylvester TT, Parsons SD, Millar RP, Helden PDV, Miller MA (2019) Performance of the tuberculin skin test in Mycobacterium bovis exposed and unexposed african lions (Panthera leo). J Wild Dis 55:537-543

14. Miller RS, Sweeney SJ (2013) Mycobacterium bovis (bovine tuberculosis) infection in North American wildlife: current status and opportunities for mitigation of risks of further infection in wildlife populations. Epidemiol Infect 141:1357-1370

15. Maciel ALG, Loiko MR, Bueno TS, Moreira JG, Coppola M, Dalla Costa ER, Schmid KB, Rodrigues RO, Cibulski SP, Bertagnolli AC, Mayer FQ (2018) Tuberculosis in Southern Brazilian wild boars (Sus scrofa): first epidemiological findings. Transbound Emerg Dis 65:518-526

16. Buddle BM, Aldwell FE, Pfeffer A, De Lisle GW (1994) Experimental Mycobacterium bovis infection in the brushtail possum (Trichosurus vulpecula): pathology, haematology and lymphocyte stimulation responses. Vet Microbiol 38:241-254

17. WAHIS Interface (2019) Animal Health Information. https://www.oie.int/ wahis_2/public/wahid.php/Diseaseinformation/statuslist. Accessed 19 Nov 2020.

18. Ryser-Degiorgis MP (2013) Wildlife health investigations: needs, challenges and recommendations. BMC Vet Res 9:223

19. Thomas J, Risalde MÁ, Serrano M, Sevilla I, Geijo M, Ortíz JA, Fuertes M, Ruíz-Fons JF, de la Fuente J, Domínguez L, Juste R (2017) The response of red deer to oral administration of heat-inactivated Mycobacterium bovis and challenge with a field strain. Vet Microbiol 208:195-202

20. Balseiro A, Prieto JM, Álvarez V, Lesellier S, Davé D, Salguero FJ, Sevilla IA, Infantes-Lorenzo JA, Garrido JM, Adriaensen H, Juste RA (2020) Protective effect of oral BCG and inactivated Mycobacterium bovis vaccines in European badgers (Meles meles) experimentally infected with M. bovis. Front Vet Sci 7:41

21. Jaroso R, Vicente J, Martín-Hernando MP, Aranaz A, Lyashchenko K, Greenwald R, Esfandiari J, Gortázar C (2010) Ante-mortem testing wild fallow deer for bovine tuberculosis. Vet Microbiol 146:285-289

22. Jaroso R, Vicente J, Fernandez-de-Mera IG, Aranaz A, Gortázar C (2010) Eurasian wild boar response to skin-testing with mycobacterial and non-mycobacterial antigens. Prev Vet Med 96:211-217

23. Risalde MÁ, Thomas J, Sevilla I, Serrano M, Ortíz JA, Garrido J, Domínguez M, Domínguez L, Gortázar C, Ruíz-Fons JF (2017) Development and evaluation of an interferon gamma assay for the diagnosis of 
tuberculosis in red deer experimentally infected with Mycobacterium bovis. BMC Vet Res 13:341

24. Thomas J, Infantes-Lorenzo JA, Moreno I, Romero B, Garrido JM, Juste R, Dominguez M, Dominguez L, Gortazar C, Risalde MA (2019) A new test to detect antibodies against Mycobacterium tuberculosis complex in red deer serum. Vet J 244:98-103

25. Thomas J, Infantes-Lorenzo JA, Moreno I, Cano-Terriza D, de Juan L, García-Bocanegra I, Domínguez L, Domínguez M, Gortázar C, Risalde MA (2019) Validation of a new serological assay for the identification of Mycobacterium tuberculosis complex-specific antibodies in pigs and wild boar. Prev Vet Med 162:11-17

26. Chambers MA (2009) Review of the diagnosis and study of tuberculosis in non-bovine wild life species using immunological methods. Transbound Emerg Dis 56:215-227

27. Chambers MA (2013) Review of the diagnosis of tuberculosis in nonbovid wildlife species using immunological methods-an update of published work since 2009. Transbound Emerg Dis 60:14-27

28. Gavier-Widen D, Cooke MM, Gallagher J, Chambers MA, Gortázar C (2009) A review of infection of wildlife hosts with Mycobacterium bovis and the diagnostic difficulties of the 'no visible lesion'presentation. N Z Vet J 57:122-131

29. Maas M, Michel AL, Rutten VP (2013) Facts and dilemmas in diagnosis of tuberculosis in wildlife. Comp Immunol Microbiol Infect Dis 36:269-285

30. Buddle BM, de Lisle GW, Griffin JFT, Hutchings SA (2015) Epidemiology, diagnostics, and management of tuberculosis in domestic cattle and deer in New Zealand in the face of a wildlife reservoir. N Z Vet J 63:19-27

31. Moher D, Shamseer L, Clarke M, Ghersi D, Liberati A, Petticrew M, Shekelle P, Stewart LA (2015) Preferred reporting items for systematic review and meta-analysis protocols (PRISMA-P) 2015 statement. Syst Rev 4:1

32. Krebs JR, Anderson R, Clutton-Brock T, Morrison I, Young D, Donnelly C, Frost S, Woodroffe R (1997) Bovine tuberculosis in cattle and badgers: report to the Rt Hon Dr. Jack Cunningham MP. Her Majesty's Stationary Office, London

33. Murphy D, Gormley E, Costello E, O'Meara D, Corner LA (2010) The prevalence and distribution of Mycobacterium bovis infection in European badgers (Meles meles) as determined by enhanced post mortem examination and bacteriological culture. Res Vet Sci 88:1-5

34. Corner LAL, Murphy D, Gormley E (2011) Mycobacterium bovis infection in the Eurasian badger (Meles meles): the disease, pathogenesis, epidemiology and control. J Comp Pathol 144:1-24

35. Buddle BM, Wedlock DN, Denis M (2006) Progress in the development of tuberculosis vaccines for cattle and wild life. Vet Microbiol 112:191-200

36. Garrido JM, Sevilla IA, Beltrán-Beck B, Minguijón E, Ballesteros C, Galindo RC, Boadella M, Lyashchenko KP, Romero B, Geijo MV, Ruiz-Fons F (2011) Protection against tuberculosis in Eurasian wild boar vaccinated with heat-inactivated Mycobacterium bovis. PLoS One 6:e24905

37. Fitzgerald SD, Kaneene JB (2013) Wildlife reservoirs of bovine tuberculosis worldwide: hosts, pathology, surveillance, and control. Vet Pathol 50:488-499

38. Gallagher J, Clifton-Hadley RS (2000) Tuberculosis in badgers; a review of the disease and its significance for other animals. Res Vet Sci 69:203-217

39. Cardoso-Toset F, Gomez-Laguna J, Amarilla SP, Vela Al, Carrasco L, Fernandez-Garayzabal JF, Astorga RJ, Luque I (2015) Multi-etiological nature of tuberculosis-like lesions in condemned pigs at the slaughterhouse. PLoS One 10:e0139130

40. Santos N, Geraldes M, Afonso A, Almeida V, Correia-Neves M (2010) Diagnosis of tuberculosis in the wild boar (Sus scrofa): a comparison of methods applicable to hunter-harvested animals. PLoS One 5:e12663

41. Davis JM, Ramakrishnan L (2009) The role of the granuloma in expansion and dissemination of early tuberculous infection. Cell 136:37-49

42. Gil O, Díaz I, Vilaplana C, Tapia G, Díaz J, Fort M, Cáceres N, Pinto S, Caylà J, Corner L, Domingo M (2010) Granuloma encapsulation is a key factor for containing tuberculosis infection in minipigs. PLoS One 5:e10030

43. Balseiro A, Rodríguez O, González-Quirós P, Merediz I, Sevilla IA, Davé D, Dalley DJ, Lesellier S, Chambers MA, Bezos J, Muñoz M, Delahay RJ, Gortázar C, Prieto JM (2011) Infection of Eurasian badgers (Meles meles) with Mycobacterium bovis and Mycobacterium avium complex in Spain. Vet J 190:e21-25
44. Viljoen IM, Van Helden PD, Millar RP (2015) Mycobacterium bovis infection in the lion (Panthera leo): current knowledge, conundrums and research challenges. Vet Microbiol 177:252-260

45. Sumi S, Radhakrishnan VV (2009) Evaluation of immunohistochemistry with a panel of antibodies against recombinant mycobacterial antigens for the diagnosis of tuberculous lymphadenitis. Int J Med Med Sci 1:215-219

46. Domingos SC, Júnior HRC, Lilenbaum W, Santa Rosa MT, Pereira CD, Medeiros LS (2019) A systematic review on the distribution of Mycobacterium bovis infection among wildlife in the Americas. Trop Anim Health Prod 51:1801-1805

47. Miller M, Buss P, Hofmeyr J, Olea-Popelka F, Parsons S, van Helden P (2015) Antemortem diagnosis of Mycobacterium bovis infection in freeranging African lions (Panthera leo) and implications for transmission. J Wildl Dis 51:493-497

48. Mikota SK, Gairhe K, Giri K, Hamilton K, Miller M, Paudel S, Lyashchenko K, Larsen RS, Payeur JB, Waters WR, Greenwald R (2015) Tuberculosis surveillance of elephants (Elephas maximus) in Nepal at the captivewild interface. Eur J Wildl Res 61:221-229

49. Crawshaw TR, Griffiths IB, Clifton-Hadley RS (2008) Comparison of a standard and a detailed postmortem protocol for detecting Mycobacterium bovis in badgers. Vet Rec 163:473-477

50. Aranaz A, Liébana E, Gómez-Mampaso E, Galán JC, Cousins D, Ortega A, Blázquez J, Baquero F, Mateos A, Súarez G, Domínguez L (1999) Mycobacterium tuberculosis subsp. caprae subsp. nov.: a taxonomic study of a new member of the Mycobacterium tuberculosis complex isolated from goats in Spain. Int J Syst Evol Microbiol 49:1263-1273

51. Cedeño I, de Obaldía R, Sanjur O, Bayard V, Ortega-Barría E, Escobar C (2005) Use of the polymerase chain reaction for diagnosing bovine tuberculosis in Panama. Rev Sci Technol 24:1067

52. Balseiro A, González-Quirós P, Rodríguez Ó, Copano MF, Merediz I, de Juan L, Chambers MA, Delahay RJ, Marreros N, Royo LJ, Bezos J (2013) Spatial relationships between Eurasian badgers (Meles meles) and cattle infected with Mycobacterium bovis in Northern Spain. Vet J 197:739-745

53. Milian-Suazo F, Garcia-Casanova L, Robbe-Austerman S, Canto-Alarcon GJ, Barcenas-Reyes I, Stuber T, Rodriguez-Hernandez E, Flores-Villalva S (2016) Molecular relationship between strains of M. bovis from Mexico and those from countries with free trade of cattle with Mexico. PLoS One 11:0155207

54. Carvalho RCT, Vasconcellos SEG, de Azevedo IM, Soares Filho PM, Mota PMPC, de Araújo FR, da Silva Carvalho AC, Gomes HM, Suffys PN, de Souza Figueiredo EE, Paschoalin VMF (2016) Molecular typing of Mycobacterium bovis from cattle reared in Midwest Brazil. PLoS One 11:0162459

55. Waters WR, Palmer MV, Bannantine JP, Greenwald R, Esfandiari J, Andersen P, McNair J, Pollock JM, Lyashchenko KP (2005) Antibody responses in reindeer (Rangifer tarandus) infected with Mycobacterium bovis. Clin Diagn Lab Immun 12:727-735

56. Wiker HG (2009) MPB70 and MPB83 - major antigens of Mycobacterium bovis. Scand I Immunol 69:492-499

57. Vordermeier HM, Whelan A, Cockle PJ, Farrant L, Palmer N, Hewinson RG (2001) Use of synthetic peptides derived from the antigens ESAT-6 and CFP-10 for differential diagnosis of bovine tuberculosis in cattle. Clin Diagn Lab Immun 8:571-578

58. Jones GJ, Hewinson RG, Vordermeier HM (2010) Screening of predicted secreted antigens from Mycobacterium bovis identifies potential novel differential diagnostic reagents. Clin Vaccine Immunol 17:1344-1348

59. Infantes-Lorenzo JA, Moreno I, de los Ángeles Risalde M, Roy Á, Villar M, Romero B, Ibarrola N, de la Fuente J, Puentes E, de Juan L, Gortázar C (2017) Proteomic characterisation of bovine and avian purified protein derivatives and identification of specific antigens for serodiagnosis of bovine tuberculosis. Clin Proteom 14:36

60. Dannenberg AM Jr (1991) Delayed-type hypersensitivity and cellmediated immunity in the pathogenesis of tuberculosis. Immunol Today 12:228-233

61. Palmer MV, Whipple DL, Waters WR (2001) Tuberculin skin testing in white-tailed deer (Odocoileus virginianus). J Vet Diagn Invest 13:530-533

62. Keet DF, Michel AL, Bengis RG, Becker P, Van Dyk DS, Van Vuuren M, Rutten VPMG, Penzhorn BL (2010) Intradermal tuberculin testing of wild African lions (Panthera leo) naturally exposed to infection with Mycobacterium bovis. Vet Microbiol 144:384-391 
63. Parsons SD, Cooper D, McCall AJ, McCall WA, Streicher EM, le Maitre NC, Müller A, van Pittius NCG, Warren RM, van Helden PD (2011) Modification of the QuantiFERON-TB Gold (In-Tube) assay for the diagnosis of Mycobacterium bovis infection in African buffaloes (Syncerus caffer). Vet Immunol Immunopathol 142:113-118

64. Mahmood KH, Stanford JL, Rook GAW, Stuart FA, Pritchard DG, Brewer Jl (1987) The immune response in two populations of wild badgers naturally infected with bovine tubercle bacilli. Tubercle 68:1 19-125

65. Mikota SK, Peddie L, Peddie J, Isaza R, Dunker F, West G, Lindsay W, Larsen RS (2001) Epidemiology and diagnosis of Mycobacterium tuberculosis in captive Asian elephants (Elephas maximus). J Wildl Dis 32:1-16

66. Clarke C, Cooper D, Goosen WJ, McFadyen R, Warren RM, van Helden PD, Parsons SDC, Miller MA (2018) Antigen-specific interferon-gamma release is decreased following the single intradermal comparative cervical skin test in African buffaloes (Syncerus caffer). Vet Immunol Immunopathol 201:12-15

67. Bouts T, Vordermeier M, Flach E, Routh A (2009) Positive skin and serologic test results of diagnostic assays for bovine tuberculosis and subsequent isolation of Mycobacterium interjectum in a pygmy hippopotamus (Hexaprotodon liberiensis). J Zoo Wildl Med 40:536-542

68. Griffin JF, Nagai S, Buchan GS (1991) Tuberculosis in domesticated red deer: comparison of purified protein derivative and the specific protein MPB70 for in vitro diagnosis. Res Vet Sci 50:279-285

69. Palmer MV, Whipple DL, Payeur JB, Bolin CA (2011) Use of the intradermal tuberculin test in a herd of captive elk (Cervus elaphus nelsoni) naturally infected with Mycobacterium bovis. J Vet Diagn Invest 23:363-366

70. Munang'andu HM, Siamudaala V, Matandiko W, Nambota A, Muma JB, Mweene AS, Munyeme M (2011) Comparative intradermal tuberculin testing of free-ranging African buffaloes (Syncerus caffer) captured for ex situ conservation in the Kafue basin ecosystem in Zambia. Vet Med Int. https://doi.org/10.4061/2011/385091

71. Bernitz N, Goosen WJ, Clarke C, Kerr TJ, Higgitt R, Roos EO, Cooper DV, Warren RM, van Helden PD, Parsons SD, Miller MA (2018) Parallel testing increases detection of Mycobacterium bovis-infected African buffaloes (Syncerus caffer). Vet Immunol Immunopathol 204:40-43

72. Gerace E, Mandanici F, Pasquali P, Falduto M, Vitale M, Presti VDML, Amato B (2018) M. bovis infection in pigs: improvement of the $\gamma$-IFN assay efficiency in this species using a maintenance medium. Tuberculosis 108:151-154

73. Angkawanish T, Morar D, Van Kooten P, Bontekoning I, Schreuder J, Maas M, Wajjwalku W, Sirimalaisuwan A, Michel A, Tijhaar E, Rutten VPMG (2013) The elephant interferon gamma assay: a contribution to diagnosis of tuberculosis in elephants. Transbound Emerg Dis 60:53-59

74. Buddle BM, Skinner MA, Chambers MA (2000) Immunological approaches to the control of tuberculosis in wildlife reservoirs. Vet Immunol Immunopathol 74:1-16

75. Harrington NP, Surujballi OP, Prescott JF, Duncan JR, Waters WR, Lyashchenko K, Greenwald R (2008) Antibody responses of cervids (Cervus elaphus) following experimental Mycobacterium bovis infection and the implications for immunodiagnosis. Clin Vaccine Immunol 15:1650-1658

76. Fernández-de-Mera IG, Vicente J, Höfle U, Fons FR, Ortiz JA, Gortázar C (2009) Factors affecting red deer skin test responsiveness to bovine and avian tuberculin and to phytohaemagglutinin. Prev Vet Med 90:119-126

77. Queirós J, Alvarez J, Carta T, Mateos A, Ortiz JA, Fernandez-de-Mera IG, Martín-Hernando MP, Gortázar C (2012) Unexpected high responses to tuberculin skin-test in farmed red deer: implications for tuberculosis control. Prev Vet Med 104:327-334

78. Che-Amat A, Risalde MÁ, González-Barrio D, Ortíz JA, Gortázar C (2016) Effects of repeated comparative intradermal tuberculin testing on test results: a longitudinal study in TB-free red deer. BMC Vet Res 12:184

79. Waters WR, Palmer MV, Thacker TC, Orloski K, Nol P, Harrington NP, Olsen SC, Nonnecke BJ (2008) Blood culture and stimulation conditions for the diagnosis of tuberculosis in cervids by the Cervigam assay. Vet Rec 162:203-208

80. Dalley D, Davé D, Lesellier S, Palmer S, Crawshaw T, Hewinson RG, Chambers M (2008) Development and evaluation of a gammainterferon assay for tuberculosis in badgers (Meles meles). Tuberculosis 88:235-243

81. Michel AL, Cooper D, Jooste J, De Klerk LM, Jolles A (2011) Approaches towards optimising the gamma interferon assay for diagnosing
Mycobacterium bovis infection in African buffalo (Syncerus caffer). Prev Vet Med 98:142-151

82. Pesciaroli M, Russo M, Mazzone P, Aronica V, Fiasconaro M, Boniotti MB, Corneli S, Cagiola M, Pacciarini M, Di Marco V, Pasquali P (2012) Evaluation of the interferon-gamma (IFN- $\gamma$ ) assay to diagnose Mycobacterium bovis infection in pigs. Vet Immunol Immunopathol 148:369-372

83. Palmer MV, Waters WR, Whipple DL, Slaughter RE, Jones SL (2004) Evaluation of an in vitro blood-based assay to detect production of interferon- $\gamma$ by Mycobacterium Bovis infected white-tailed Deer (Odocoileus Virginianus). J Vet Diagn Invest 16:17-21

84. Harrington NP, Surujballi OP, Waters WR, Prescott JF (2007) Development and evaluation of a real-time reverse transcription-PCR assay for quantification of gamma interferon mRNA to diagnose tuberculosis in multiple animal species. Clin Vaccine Immunol 14:1563-1571

85. Goosen WJ, Miller MA, Chegou NN, Cooper D, Warren RM, van Helden PD, Parsons SD (2014) Agreement between assays of cell-mediated immunity utilizing Mycobacterium bovis-specific antigens for the diagnosis of tuberculosis in African buffaloes (Syncerus caffer). Vet Immunol Immunopathol 160:133-138

86. Goosen WJ, Cooper D, Miller MA, van Helden PD, Parsons SD (2015) IP-10 is a sensitive biomarker of antigen recognition in whole-blood stimulation assays used for the diagnosis of Mycobacterium bovis infection in African buffaloes (Syncerus caffer). Clin Vaccine Immunol 22:974-978

87. Goosen WJ, van Helden PD, Warren RM, Miller MA, Parsons SD (2016) The stability of plasma IP-10 enhances its utility for the diagnosis of Mycobacterium bovis infection in African buffaloes (Syncerus caffer). Vet Immunol Immunopathol 173:17-20

88. Bernitz N, Kerr TJ, Goosen WJ, Clarke C, Higgitt R, Roos EO, Cooper DV, Warren RM, van Helden PD, Parsons SD, Miller MA (2019) Parallel measurement of IFN- $y$ and IP-10 in QuantiFERON ${ }^{\circledR}$-TB Gold (QFT) plasma improves the detection of Mycobacterium bovis infection in African buffaloes (Syncerus caffer). Prev Vet Med 169:104700

89. Roos EO, Olea-Popelka F, Buss P, de Klerk-Lorist LM, Cooper D, Warren RM, van Helden PD, Parsons SD, Miller MA (2018) IP-10: a potential biomarker for detection of Mycobacterium bovis infection in warthogs (Phacochoerus africanus). Vet Immunol Immunopathol 201:43-48

90. Clarke C, Patterson SJ, Drewe JA, Van Helden PD, Miller MA, Parsons SDC (2016) Development and evaluation of a diagnostic cytokine-release assay for Mycobacterium suricattae infection in meerkats (Suricata suricatta). BMC Vet Res 13:2

91. Surujballi OM, Lutze-Wallace C, Turcotte C, Savic M, Stevenson D, Romanowska A, Monagle W, Berlie-Surujballi G, Tangorra E (2009) Sensitive diagnosis of bovine tuberculosis in a farmed cervid herd with use of an MPB70 protein fluorescence polarization assay. Can J Vet Res 73:161

92. Dalley D, Chambers MA, Cockle P, Pressling W, Gavier-Widen D, Hewinson RG (1999) A lymphocyte transformation assay for the detection of Mycobacterium bovis infection in the Eurasian badger (Meles meles). Vet Immunol Immunopathol 70:85-94

93. Sawyer J, Mealing D, Dalley D, Dave D, Lesellier S, Palmer S, BowenDavies J, Crawshaw TR, Chambers MA (2007) Development and evaluation of a test for tuberculosis in live European badgers (Meles meles) based on measurement of gamma interferon mRNA by real-time PCR. J Clin Microbiol 45:2398-2403

94. McNair J, Welsh MD, Pollock JM (2007) The immunology of bovine tuberculosis and progression toward improved disease control strategies. Vaccine 25:5504-5511

95. Kampfer S, Dalley D, Hewinson RG, Chambers MA, Singh M (2003) Multi-antigen ELISA for enhanced diagnosis of tuberculosis in badgers. Vet Rec 153:403-403

96. Chambers MA, Waterhouse S, Lyashchenko K, Delahay R, Sayers R, Hewinson RG (2009) Performance of TB immunodiagnostic tests in Eurasian badgers (Meles meles) of different ages and the influence of duration of infection on serological sensitivity. BMC Vet Res 5:42

97. Roos EO, Buss P, de Klerk-Lorist LM, Hewlett J, Hausler GA, Rossouw L, McCall AJ, Cooper D, van Helden PD, Parsons SD, Miller MA (2016) Test performance of three serological assays for the detection of Mycobacterium bovis infection in common warthogs (Phacochoerus africanus). Vet Immunol Immunopathol 182:79-84 
98. Larsen RS, Salman MD, Mikota SK, Isaza R, Montali RJ, Triantis J (2000) Evaluation of a multiple-antigen enzyme-linked immunosorbent assay for detection of Mycobacterium tuberculosis infection in captive elephants. J Wildl Dis 31:291-302

99. Infantes-Lorenzo JA, Dave D, Moreno I, Anderson P, Lesellier S, Gormley E, Dominguez L, Balseiro A, Gortázar C, Dominguez M, Salguero FJ (2019) New serological platform for detecting antibodies against Mycobacterium tuberculosis complex in European badgers. Vet Med Sci 5:61-69

100. Buddle BM, Nolan A, McCarthy AR, Heslop J, Aldwell FE, Jackson R, Pfeiffer DU (1995) Evaluation of three serological assays for the diagnosis of Mycobacterium bovis infection in brushtail possums. N Z Vet J 43:91-95

101. Greenwald R, Esfandiari J, Lesellier S, Houghton R, Pollock J, Aagaard C, Andersen P, Hewinson RG, Chambers M, Lyashchenko K (2003) Improved serodetection of Mycobacterium bovis infection in badgers (Meles meles) using multiantigen test formats. Diagn Microbiol Infect Dis 46:197-203

102. Wadhwa A, Johnson RE, Mackintosh CG, Griffin JFT, Waters WR, Bannantine JP, Eda S (2013) Use of ethanol extract of Mycobacterium bovis for detection of specific antibodies in sera of farmed red deer (Cervus elaphus) with bovine tuberculosis. BMC Vet Res 9:256

103. Surujballi OP, Romanowska A, Sugden EA, Turcotte C, Jolley ME (2002) A fluorescence polarization assay for the detection of antibodies to Mycobacterium bovis in cattle sera. Vet Microbiol 87:149-157

104. Shury TK, Bergeson D, Surujballi O, Lyashchenko KP, Greenwald R (2014) Field evaluation of three blood-based assays for elk (Cervus canadensis) naturally infected with Mycobacterium bovis. Prev Vet Med 115:109-121

105. Chapinal N, Schumaker BA, Joly DO, Elkin BT, Stephen C (2015) Bayesian analysis to evaluate tests for the detection of Mycobacterium bovis infection in free-ranging wild bison (bison bison athabascae) in the absence of a gold standard. J Wildl Dis 51:619-625

106. Lyashchenko KP, Singh M, Colangeli R, Gennaro ML (2000) A multi-antigen print immunoassay for the development of serological diagnosis of infectious diseases. J Immunol Methods 242:91-100

107. Waters W, Stevens GE, Schoenbaum MA, Orloski KA, Robbe-Austerman S, Harris NB, Hall SM, Thomsen BV, Wilson AJ, Brannian RE, Nelson JT (2011) Bovine tuberculosis in a Nebraska herd of farmed elk and fallow deer: a failure of the tuberculin skin test and opportunities for serodiagnosis. Vet Med Int 953985

108. Lyashchenko KP, Gortázar C, Miller MA, Waters WR (2018) Spectrum of antibody profiles in tuberculous elephants, cervids, and cattle. Vet Microbiol 214:89-92

109. Lyashchenko KP, Greenwald R, Esfandiari J, Chambers MA, Vicente J, Gortazar C, Santos N, Correia-Neves M, Buddle BM, Jackson R, O'Brien DJ (2008) Animal-side serologic assay for rapid detection of Mycobacterium bovis infection in multiple species of free-ranging wildlife. Vet Microbiol 132:283-292

110. Miller MA, Gortazar C, Roos EO, Risalde MA, Johnathan-Lee A, Sridhara AA, Lyashchenko KP (2019) Serological reactivity to MPB83 and CFP10/ ESAT-6 antigens in three suid hosts of Mycobacterium bovis infection. Vet Microbiol 235:285-288

111. Himsworth CG, Elkin BT, Nishi JS, Epp T, Lyashchenko KP, Surujballi O, Turcotte C, Esfandiari J, Greenwald R, Leighton FA (2010) Comparison of test performance and evaluation of novel immunoassays for tuberculosis in a captive herd of wood bison naturally infected with Mycobacterium bovis. J Wildl Dis 46:78-86

112. Duncan AE, Lyashchenko K, Greenwald R, Miller M, Ball R (2009) Application of Elephant TB STAT-PAK assay and MAPIA (multi-antigen print immunoassay) for detection of tuberculosis and monitoring of treatment in black rhinoceros (Diceros bicornis). J Zoo Wildl Med 40:781-785

113. O'Brien DJ, Schmitt SM, Lyashchenko KP, Waters WR, Berry DE, Palmer MV, McNair J, Greenwald R, Esfandiari J, Cosgrove MK (2009) Evaluation of blood assays for detection of Mycobacterium bovis in white-tailed deer (Odocoileus virginianus) in Michigan. J Wild Dis 45:153-164

114. Michel AL, Simões M (2009) Comparative field evaluation of two rapid immunochromatographic tests for the diagnosis of bovine tuberculosis in African buffaloes (Syncerus caffer). Vet Immunol Immunopathol 127:186-189

115. Miller M, Joubert J, Mathebula N, De Klerk-Lorist LM, Lyashchenko KP, Bengis R, van Helden P, Hofmeyr M, Olea-Popelka F, Greenwald R,
Esfandiari J (2012) Detection of antibodies to tuberculosis antigens in free-ranging lions (Panthera leo) infected with Mycobacterium bovis in Kruger National Park, South Africa. J Wildl Dis 43:317-323

116. Brüns AC, Tanner M, Williams MC, Botha L, O'Brien A, Fosgate GT, Van Helden PD, Clarke J, Michel AL (2017) Diagnosis and implications of Mycobacterium bovis infection in banded mongooses (Mungos mungo) in the Kruger National Park, South Africa. J Wildl Dis 53:19-29

117. Gowtage-Sequeira S, Paterson A, Lyashchenko KP, Lesellier S, Chambers MA (2009) Evaluation of the CervidTB STAT-PAK for the detection of Mycobacterium bovis infection in wild deer in Great Britain. Clin Vaccine Immunol 16:1449-1452

118. Rosen LE, Hanyire TG, Dawson J, Foggin CM, Michel AL, Huyvaert KP, Miller MA, Olea-Popelka FJ (2018) Tuberculosis serosurveillance and management practices of captive African elephants (Loxodonta africana) in the Kavango-Zambezi Transfrontier Conservation Area. Transbound Emerg Dis 65:e344-e354

119. Buddle BM, Wilson T, Denis M, Greenwald R, Esfandiari J, Lyashchenko KP, Liggett S, Mackintosh CG (2010) Sensitivity, specificity, and confounding factors of novel serological tests used for the rapid diagnosis of bovine tuberculosis in farmed red deer (Cervus elaphus). Clinl Vaccine Immunol 17:626-630

120. Boadella M, Barasona JA, Diaz-Sanchez S, Lyashchenko KP, Greenwald R, Esfandiari J, Gortazar C (2012) Performance of immunochromatographic and ELISA tests for detecting fallow deer infected with Mycobacterium bovis. Prev Vet Med 104:160-164

121. Lyashchenko KP, Greenwald R, Esfandiari J, O'Brien DJ, Schmitt SM, Palmer MV, Waters WR (2013) Rapid detection of serum antibody by dual-path platform VetTB assay in white-tailed deer infected with Mycobacterium bovis. Clin Vaccine Immunol 20:907-911

122. Lyashchenko KP, Greenwald R, Esfandiari J, Mikota S, Miller M, Moller T, Vogelnest L, Gairhe KP, Robbe-Austerman S, Gai J, Waters WR (2012) Field application of serodiagnostics to identify elephants with tuberculosis prior to case confirmation by culture. Clin Vaccine Immunol 19:1269-1275

123. Courcier EA, Pascual-Linaza AV, Arnold ME, McCormick CM, Corbett DM, O'Hagan MJH, Collins SF, Trimble NA, McGeown CF, McHugh GE, McBride KR (2020) Evaluating the application of the dual path platform VetTB test for badgers (Meles meles) in the test and vaccinate or remove (TVR) wildlife research intervention project in Northern Ireland. Res Vet Sci 130:170-178

124. Che'Amat A, González-Barrio D, Ortiz JA, Díez-Delgado I, Boadella M, Barasona JA, Bezos J, Romero B, Armenteros JA, Lyashchenko KP, Venteo A (2015) Testing Eurasian wild boar piglets for serum antibodies against Mycobacterium bovis. Prev Vet Med 121:93-98

125. Fresco-Taboada A, Risalde MA, Gortázar C, Tapia I, González I, Venteo Á, Sanz A, Rueda P (2019) A lateral flow assay for the rapid diagnosis of Mycobacterium bovis infection in wild boar. Transbound Emerg Dis $66: 2175-2179$

126. Griffin JFT, Cross JP, Chinn DN, Rodgers CR, Buchan GS (1994) Diagnosis of tuberculosis due to Mycobacterium bovis in New Zealand red deer (Cervus elaphus) using a composite blood test and antibody assays. N Z Vet J 42:173-179

127. Olivier TT, Viljoen IM, Hofmeyr J, Hausler GA, Goosen WJ, Tordiffe ASW, Buss P, Loxton AG, Warren RM, Miller MA, van Helden PD (2017) Development of a gene expression assay for the diagnosis of Mycobacterium bovis infection in African lions (Panthera leo). Transbound Emerg Dis 64:774-781

128. Fend R, Geddes R, Lesellier S, Vordermeier HM, Corner LAL, Gormley E, Costello E, Hewinson RG, Marlin DJ, Woodman AC, Chambers MA (2005) Use of an electronic nose to diagnose Mycobacterium bovis infection in badgers and cattle. J Clin Microbiol 43:1745-1751

129. Stahl RS, Ellis CK, Nol P, Waters WR, Palmer M, VerCauteren KC (2015) Fecal volatile organic compound profiles from white-tailed deer (Odocoileus virginianus) as indicators of Mycobacterium bovis exposure or Mycobacterium bovis bacille calmette-guerin (BCG) vaccination. PLoS One 10:e0129740

130. Nol P, Ionescu R, Geremariam Welearegay T, Barasona JA, Vicente J, de Jesus B-Sáenz K, Barrenetxea I, Jose Torres M, Ionescu F, Rhyan J (2020) Evaluation of volatile organic compounds obtained from breath and feces to detect Mycobacterium tuberculosis complex in wild boar (Sus scrofa) in Doñana National Park, Spain. Pathogens 9:346 
131. Fernández-de-Mera IG, Vicente J, Höfle U, Rodriguez O, Gaspar-Lopez E, Gortázar C (2008) The effects of sex and age on phytohaemagglutinin skin-testing of deer. N Z Vet J 56:71-73

132. Fernández-de-Mera IG, Jaroso R, Martín-Hernando MP, Queirós J, Carta T, Ortiz JA, Vicente J, Gortázar C (2011) The testing season affects red deer skinfold increase in response to phytohaemagglutinin. Prev Vet Med 100:79-83

133. Boadella M, Lyashchenko K, Greenwald R, Esfandiari J, Jaroso R, Carta T, Garrido JM, Vicente J, de la Fuente J, Gortázar C (2011) Serologic tests for detecting antibodies against Mycobacterium bovis and Mycobacterium avium subspecies paratuberculosis in Eurasian wild boar (Sus scrofa scrofa). J Vet Diagn Invest 23:77-83

134. Gcebe N, Hlokwe TM (2017) Non-tuberculous mycobacteria in South African wildlife: neglected pathogens and potential impediments for bovine tuberculosis diagnosis. Front Cell Infect Microbiol 7:15

135. Palmer MV, Waters WR, Thacker TC (2020) Vaccination of whitetailed deer (Odocoileus virginianus) with Mycobacterium bovis bacille Calmette-Guérin (BCG) results in positive tuberculin skin test results in a dose-dependent fashion. Res Vet Sci 129:70-73

136. Lalvani A, Pareek M (2010) Interferon gamma release assays: principles and practice. Enferm Infecc Microbiol Clin 28:245-252

137. Aurtenetxe O, Barral M, Vicente J, de la Fuente J, Gortázar C, Juste RA (2008) Development and validation of an enzyme-linked immunosorbent assay for antibodies against Mycobacterium bovis in European wild boar. BMC Vet Res 4:43

138. Kang SS, Byeon HS, Ku BK, Kim SW, Kim J, Woo J, Ahn B, Kim S, Monoldorova S, Park CH, Cho SN (2016) Seroprevalence of tuberculosis in domesticated elk (Cervus canadensis) in Korea. Res Vet Sci 107:228-232

139. King HC, Murphy A, James P, Travis E, Porter D, Sawyer J, Cork J, Delahay RJ, Gaze W, Courtenay O, Wellington EM (2015) Performance of a noninvasive test for detecting Mycobacterium bovis shedding in European badger (Meles meles) populations. J Clinl Microbiol 53:2316-2323

140. Fifis T, Costopoulos C, Corner LA, Wood PR (1992) Serological reactivity to Mycobacterium bovis protein antigens in cattle. Vet Microbiol 30:343-354

141. Van der Heijden EM, Cooper DV, Rutten VP, Michel AL (2019) Mycobacterium bovis prevalence affects the performance of a commercial serological assay for bovine tuberculosis in African buffaloes. Comp Immunol Microbiol Infect Dis 70:101369
142. Buzdugan SN, Chambers MA, Delahay RJ, Drewe JA (2016) Diagnosis of tuberculosis in groups of badgers: an exploration of the impact of trapping efficiency, infection prevalence and the use of multiple tests. Epidemiol Infect 144:1717-1727

143. Drewe JA, Tomlinson AJ, Walker NJ, Delahay RJ (2010) Diagnostic accuracy and optimal use of three tests for tuberculosis in live badgers. PLoS One 5:e11196

144. Corrin KC, Carter CE, Kissling RC, Lisle GD (1993) An evaluation of the comparative tuberculin skin test for detecting tuberculosis in farmed deer. N Z Vet J 41:12-20

145. Waters WR, Palmer MV, Olsen SC, Sacco RE, Whipple DL (2003) Immune responses of elk to Mycobacterium bovis bacillus Calmette Guerin vaccination. Vaccine 21:1518-1526

146. Palmer MV, Waters WR, Thacker TC, Stoffregen WC, Thomsen BV (2006) Experimentally induced infection of reindeer (Rangifer tarandus) with Mycobacterium bovis. J Vet Diagn Invest 18:52-60

147. Hutchings DL, Wilson SH (1995) Evaluation of lymphocyte stimulation tests for diagnosis of bovine tuberculosis in elk (Cervus elaphus). Am J Vet Res 56:27-33

148. Nelson JT, Orloski KA, Lloyd AL, Camacho M, Schoenbaum MA, RobbeAusterman S, Thomsen BV, Hall SM (2012) Evaluation of serodiagnostic assays for Mycobacterium bovis infection in elk, white-tailed deer, and reindeer in the United States. Vet Med Int 2012:563293

149. De Val B, Napp S, Velarde R, Lavín S, Cervera Z, Singh M, Allepuz A, Mentaberre G (2017) Serological follow-up of tuberculosis in a wild boar population in contact with infected cattle. Transbound Emerg Dis 64:275-283

150. Roos EO, Olea Popelka F, Buss P, de Klerk-Lorist LM, Cooper D, van Helden PD, Parsons SDC, Miller MA (2018) Seroprevalence of Mycobacterium bovis infection in warthogs (Phacochoerus africanus) in bovine tuberculosis-endemic regions of South Africa. Transbound Emerg Dis 65:1182-1189

\section{Publisher's Note}

Springer Nature remains neutral with regard to jurisdictional claims in published maps and institutional affiliations.
Ready to submit your research? Choose BMC and benefit from:

- fast, convenient online submission

- thorough peer review by experienced researchers in your field

- rapid publication on acceptance

- support for research data, including large and complex data types

- gold Open Access which fosters wider collaboration and increased citations

- maximum visibility for your research: over 100M website views per year

At BMC, research is always in progress.

Learn more biomedcentral.com/submissions 